\section{Al】102 78408 己}

NATL INST OF STANDAAOS \& TECH R.I.C.

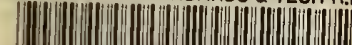

A11102784.

A11102784082
Sparks, Larry L/Thermal conductlvity of

QC100.U58 NO.88-3086 1988 V19 C. 1 NBS-

\title{
THERMAL CONDUCTIVITY OF SELECTED FOAMS AND SYSTEMS FROM 100 TO $300 \mathrm{~K}$
}

\section{L.L. Sparks}

W.P. Dubé

A.J. Slifka

National Bureau of Standards

U.S. Department of Commerce

Boulder, Colorado 80303-3328

January 1988

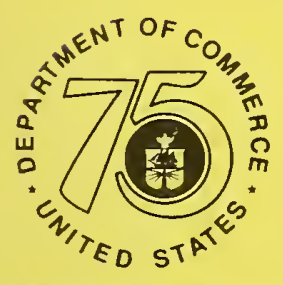

Stumulating America's Progress
1913-1988

$-Q C$

100

.456

\#88-3086

1988

C.2 



\section{THERMAL CONDUCTIVITY OF SELECTED FOAMS AND SYSTEMS FROM 100 TO $300 \mathrm{~K}$}

\section{L.L. Sparks}

W.P. Dubé

A.J. Slifka

Chemical Engineering Science Division

Center for Chemical Engineering

National Engineering Laboratory

National Bureau of Standards

Boulder, Colorado 80303-3328

January 1988

Prepared for

NASA Langley Research Center

Hampton, Virginia 23665

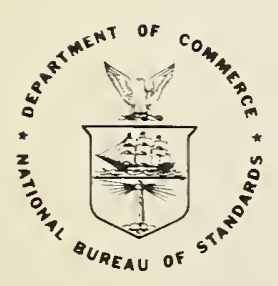

U.S. DEPARTMENT OF COMMERCE, C. William Verity, Secretary

NATIONAL BUREAU OF STANDARDS, Ernest Ambler, Director 

CONTENTS

List of Tables . . . . . . . . . . . . . . . . .

List of Figures . . . . . . . . . . . . . . . . . .

List of Units and Symbols . . . . . . . . . . . . . .

ABSTRACT

INTRODUCTION

APPARATUS

SPECIMENS . . . . . . . . . . . . . . . . . . . .

RESULTS

DISCUSSION . . . . . . . . . . . . . . . . . .

SUMMARY

REFERENCES
Page

v

vii

ix

1

1

1

5

5

32

45

46

iii 

Test specimens . . . . . . . . . . . . . 5

5 Polynomial coefficients for a four-term fit of thermal conductivity as a function of temperature for PMA51. . . . 7

6 Thermal conductivity $(\mathrm{mW} /(\mathrm{m} \cdot \mathrm{K}))$ as a function of temperature (K) from the polynomial coefficients given in table 5 for PMA5l . . . . . . . . . . . . . . . 8

8 Polynomial coefficients for a four-term fit of thermal conductivity as a function of temperature for PMA71-2. . . 11

9 Thermal conductivity $(\mathrm{mW} /(\mathrm{m} \cdot \mathrm{K}))$ as a function of temperature $(K)$ from the polynomial coefficients given in table 8 for PMA71-2................ . . 11

10 Experimental data for PMA71-1........... 14

11 Polynomial coefficients for a five-term fit of thermal conductivity as a function of temperature for PMA71-1. . 14

Thermal conductivity $(\mathrm{mW} /(\mathrm{m} \cdot \mathrm{K}))$ as a function of temperature $(K)$ from the polynomial coefficients given in table 11

for PMA71-1.............. . . 15

13 Experimental data for COMPOSITE . . . . . . . . 18

14 Polynomial coefficients for a four-term fit of thermal conductivity as a function of temperature for COMPOSITE. . 18

15 Thermal conductivity $(\mathrm{mW} /(\mathrm{m} \cdot \mathrm{K}))$ as a function of temperature (K) from the polynomial coefficients given in table 14 for COMPOSITE 
17 Polynomial coefficients for a seven-term fit of thermal conductivity as a function of temperature for an aluminum/foam composite, SOFI . . . . . . . . . .

18 Thermal conductivity $(\mathrm{mW} /(\mathrm{m} \cdot \mathrm{K}))$ as a function of temperature (K) from the polynomial coefficients given in table 17 for an alumi num/foam composite, SOFI . . . . . . . 22

19 Computed thermal conductivity of the foam component of an al uminum/foam composite, SOFI . . . . . . . . . 25

20 Polynomial coefficients for a seven-term fit of thermal conductivity as a function of temperature for the foam component of an aluminum/foam composite, SOFI . . . . 25

21 Thermal conductivity $(\mathrm{mW} /(\mathrm{m} \cdot \mathrm{K}))$ as a function of temperature $(K)$ from the coefficients given in table 20 for the foam component of an aluminum/foam composite, SOFI . . . . 26

22 Experimental data for PMA71-1-vac . . . . . . . . 29

23 Polynomial coefficients for a three-term fit of thermal conductivity as a function of temperature for PMA71-1-vac. 29

24 Thermal conductivity $(\mathrm{mW} /(\mathrm{m} \cdot \mathrm{K}))$ as a function of temperature $(K)$ from the polynomial coefficients given in table 23 for PMA71-1-vac .................

25 Computed thermal conductivity $(\mathrm{mW} /(\mathrm{m} \cdot \mathrm{K}))$ as a function of temperature (K) for a polymethacrylimide foam of density

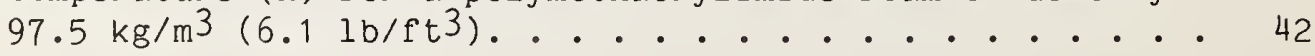

26 Thermal resistance due to evacuated boundaries, $R_{B}$ for PMS71-1 .................. 
Photo of Apparatus . . . . . . . . . . . . . 3

2 Schematic of guarded-hot-plate specimen configuration and environmental chamber

Polynomial fit of thermal conductivity $(\mathrm{mW} /(\mathrm{m} \cdot \mathrm{K}))$ as a function of temperature (K) for PMA51. Experimental data are represented by discrete points . . . . . . . . 9

4 Deviations ( $\%$ ) between experimental and fitted data as a function of temperature $(K)$ for PMA51 . . . . . . . 10

5 Polynomial fit of thermal conductivity $(\mathrm{mW} /(\mathrm{m} \cdot \mathrm{K}))$ as a function of temperature $(K)$ for PMA71-2. Experimental data are represented as discrete points . . . . . . 12

6 Deviations ( $\%$ ) between experimental and fitted data as a function of temperature $(K)$ for PMA71-2 . . . . . . 13

7 Polynomial fit of thermal conductivity $(\mathrm{mW} /(\mathrm{m} \cdot \mathrm{K})$ ) as a function of temperature $(K)$ for PMA71-1. Experimental data are represented by discrete points . . . . . . 16

8 Deviations ( $\%$ ) between experimental and fitted data as a function of temperature $(K)$ for PMA71-1. . . . . . . 17

9 Polynomial fit of thermal conductivity $(\mathrm{mW} /(\mathrm{m} \cdot \mathrm{K})$ ) as a function of temperature $(K)$ for COMPOSITE. Experimental are data represented by discrete points . . . . . . 19

10 Deviations ( $\%$ ) between experimental and fitted data as a function of temperature $(K)$ for COMPOSITE. . . . . . . 20

11 Polynomial fit of thermal conductivity $(\mathrm{mW} /(\mathrm{m} \cdot \mathrm{K}))$ as a function of temperature $(K)$ for an aluminum/foam composite, SOFI. Experimental data are represented as discrete points.............. . . 23

12 Deviations ( $\%$ ) between experimental and fitted data as a function of temperature (K) for an aluminum/foam composite, SOFI . . . . . . . . . . . . . . . . . 24

13 Polynomial fit of thermal conductivity $(\mathrm{mW} /(\mathrm{m} \cdot \mathrm{K})$ ) as a function of temperature $(K)$ for the foam component of an aluminum/foam composite, SOFI. Experimental data are represented by discrete points. 
14 Deviations (\%) between experimental and fitted data as a function of temperature $(K)$ for the foam component of an aluminum/foam composite, SOFI ............

15 Polynomial fit of thermal conductivity $(\mathrm{mW} /(\mathrm{m} \cdot \mathrm{K})$ ) as a function of temperature (K) for PMA71-1-vac. Experimental data are represented by discrete points . . . . 30

16 Deviations ( $\%$ ) of between experimental and fitted data as a function of temperature $(K)$ for PMA71-1-vac. . . . . . 31

17 (a) Possible variations of resin distribution in two foams of equal density. (b) Possible heat-flow path lengths as a function of cell geometry for two foams of equal density and for different directions in a single foam . . . . . 34

18 Fitted thermal conductivity $(\mathrm{mW} /(\mathrm{m} \cdot \mathrm{K}))$ as a function of temperature (K) for PMA51, PMA71-1, PMA71-2, COMPOSITE SOFI (PU), and PMA71-1-Vac . . . . . . . . . . 35

19 Thermal conductivity $(\mathrm{mW} /(\mathrm{m} \cdot \mathrm{K}))$ as a function of density $\left(\mathrm{kg} / \mathrm{m}^{3}\right)$ with temperature $(\mathrm{K})$ as a parameter for PMA foams. 38

20 Cross section schematic for the composite specimen . . . 39

21 Thickness effect on the measured thermal conductivity of foams (Jones, 1968). . . . . . . . . . . . . 41

22 Schematic indicating operative modes of heat transfer when the cut foam surfaces are exposed to an insulating vacuum. 


\section{Definition of Symbols}

$$
\begin{aligned}
K & =\text { thermal conductivity, } W /(m \cdot K) \\
\Delta X & =\text { mean specimen thickness, } m \\
A & =\text { area of metered section of } G H P, m^{2} \\
\Delta T & =\text { steady-state temperature difference, } K \\
Q & =\text { power }, W
\end{aligned}
$$

\section{Unit Conversions}

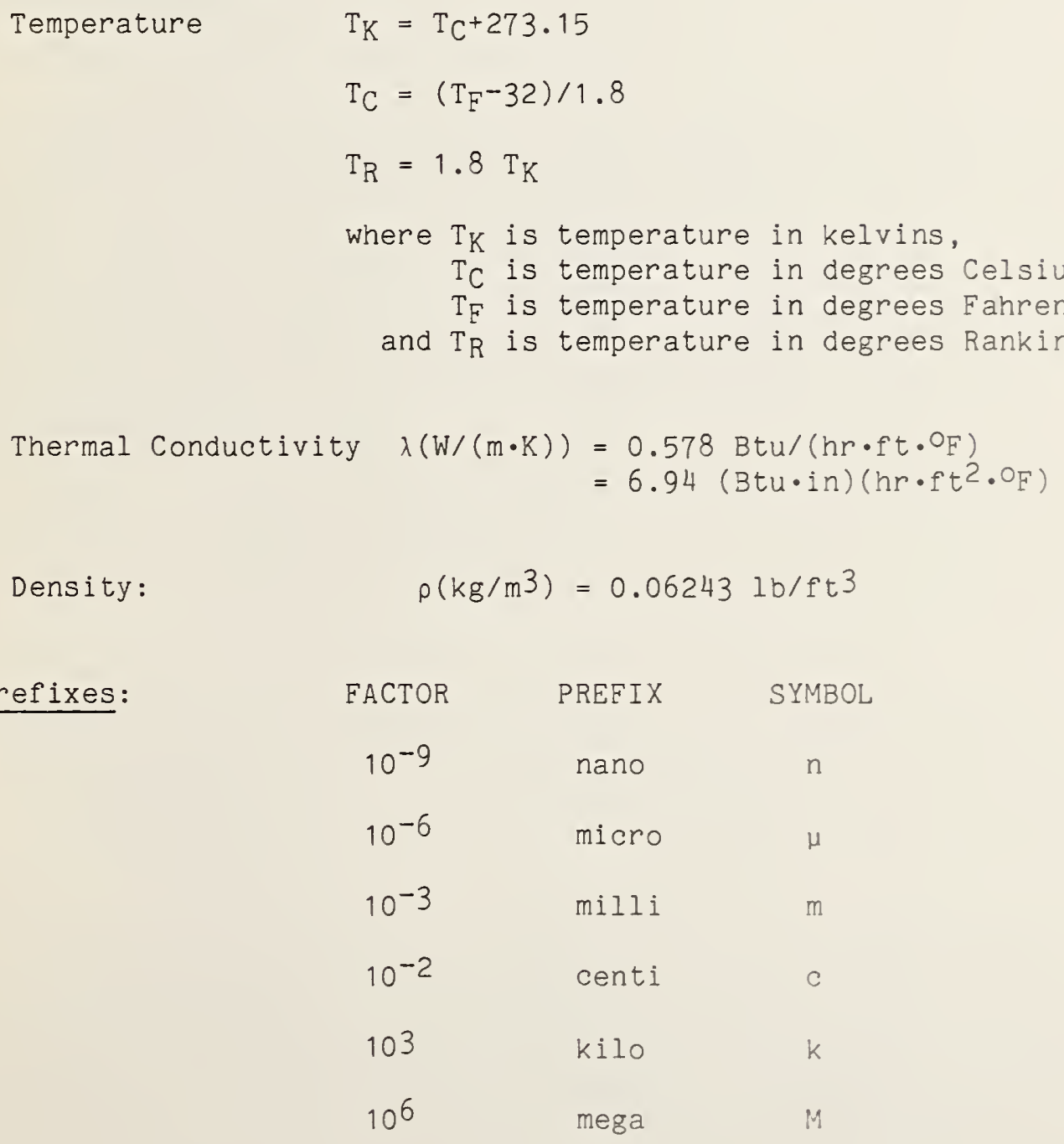





\section{Thermal Conductivity of Selected Eoams and Systems from 100 to $300 \mathrm{~K}$}

L. L. Sparks, W. P. Dube', and A. J. Slifka

Chemical Engineering Science Division

National Bureau of Standards

Boulder, Colorado 80303

The apparent thermal conductivity of five insulating materials was determined in the nominal temperature range from 100 to 300 K $\left(-280\right.$ to $\left.80 \quad O_{F}\right)$. A guarded-hot-plate apparatus was used and the testing environment was dry nitrogen gas at near ambient pressure. One specimen was retested in a vacuum environment.

Three of the specimens were neat polymethacrylimide (PUA) foams with nominal densities of 51 and $71 \mathrm{~kg} / \mathrm{m}^{3}\left(3.2\right.$ and $\left.4.4 \mathrm{lb} / \mathrm{ft}^{3}\right)$. Other specimens tested were a polyurethane foam sprayed on an aluminum substrate and a sandwich construction specimen utilizing PMA foam of nominal density $110 \mathrm{~kg} / \mathrm{m}^{3}(6.91 \mathrm{~b} / \mathrm{ft})$. The results in nitrogen gas show the expected conductivity increase with increasing density. The observed conductivity at $0.67 \mathrm{~Pa}$ ( $5 \mu \mathrm{m}$ of $\mathrm{Hg}$ ) is significantiy lower than that in ambient-pressure nitrogen gas. The large relative difference may be due to increased thermal resistance at the plate-specimen boundaries.

Key words: foam; insulation; low-temperature; polymethacrylimide; polyurethane; thermal conductivity.

\section{INTRODUCTION}

The apparent thermal conductivity of five insulating materials has been determined in the nominal temperature range from 100 to $300 \mathrm{~K}$. In each case the tests were conducted in a dry nitrogen atmosphere at a pressure slightly above ambient. One specimen was retested at a pressure of $0.67 \mathrm{~Pa}(5 \mu \mathrm{n}$ of $4 \mathrm{~g})$.

APPARATUS

The system used to make the measurements is generically known 39 . guarded-hot-plate (GHP). The NBS apparatus is pictured in figure 1 and ghom schematically in figure 2. This type of system is one of the haty stonumb Methods (ASTM, C177) for determining the apparent thermal conductivity of pos. thermal conductors. A detailed discussion of the apparatus used for thease measurements, as it existed in 1981, is given by Smith, Hust, and lan foolen (1982); the system was modified to include automated control and :a: acquisition capabilities prior to making the current experimental meajurocenet. A brief discussion of the apparatus operation is given below: 
The operation of this type of apparatus involves supplying a measured power, $Q$, from the main heater plate to the two specimens ( $\mathrm{fig}$. 2) and measuring the resulting steady-state temperature differences across the specimens. The thermal conductivity is then given by

$$
k=\frac{\Delta X}{2 A} \cdot \frac{Q}{\Delta T}
$$

where $\Delta X$ is the mean specimen thickness, $A$ is the area of the metered section of the main heater plate, and $\Delta T$ is the steady-state mean temperature difference across the two specimens. The absolute temperatures and temperature differences are measured with type $E$ thermocouples.

The accuracy of $k$ depends on the accuracy of measurement of the parameters shown in eq (1) and on establishment of unidirectional (vertical in this case) heat flow in the metered area of the specimens. The inner-guard heater plate is controlled at the temperature of the main heater in order to minimize radial heat flow in the metered area. Somers and Cyphers (1951) and ASTM C177 indicate that, when the metered diameter-to-thickness ratio of a specimen is greater than 4 , the errors in $k$ due to edge losses should be less than 1\%. The metered section diameter of the plates is $10 \mathrm{~cm}$ (3.94 in) and the specimen thicknesses are given in the SPECIMENS section.

The most difficult parameters to determine are temperature differences across the specimens, $\Delta \mathrm{T}$. The correctness of $\Delta \mathrm{T}$ measurements depends on plate-to-specimen thermal contact, on unidirectional heat flow, and on thermocouple calibration and referencing sources of error. The diverse materials which can be tested in this type of system cause a wide range of plate-to-specimen contact situations. Tye and Spinney (1976) found that embedding the thermocouple wires in the specimen, as opposed to the measuring plates, resulted in higher observed conductivities. This effect is due to better specimen-to-thermocouple contact which results in smaller measured $\Delta \mathrm{T}$ and larger $\mathrm{k}$ (eq (1)). They also found that the effect of thermocouple placement was dependent on the conductivity of the specimens. As the conductivity of the specimen increases, the relative effect of an air gap in the specimen-to-plate interface becomes larger. As discussed later in relation to the vacuum tests, specimen-to-sensor contact can strongly affect the observed conductivity.

Thermocouple placement used in obtaining the data reported below consisted of cementing the wires into machined grooves in the measuring plates (plates adjacent to the specimens shown in figure 2) so that the thermocouples were flush with the plate surfaces.

The accuracy of measurements made with this system on fiberglass and fiberboard reference materials is discussed by Smith et al. (1982). In a qualitative sense the plate-to-specimen contact should be similar for low-density foams and fibrous materials. Based on the percentage error estimate given by Smith for each parameter in eq (1) and the magnitude of these parameters for the current experiments, uncertainties in the observed values for foam are estimated to be $0.2 \mathrm{~mW} /(\mathrm{m} \cdot \mathrm{K})$ random and $0.4 \mathrm{~mW} /(\mathrm{m} \cdot \mathrm{K})$ systematic near room temperature; near $80 \mathrm{~K}$ the random and systematic components are $0.1 \mathrm{~mW} /(\mathrm{m} \cdot \mathrm{K})$. 


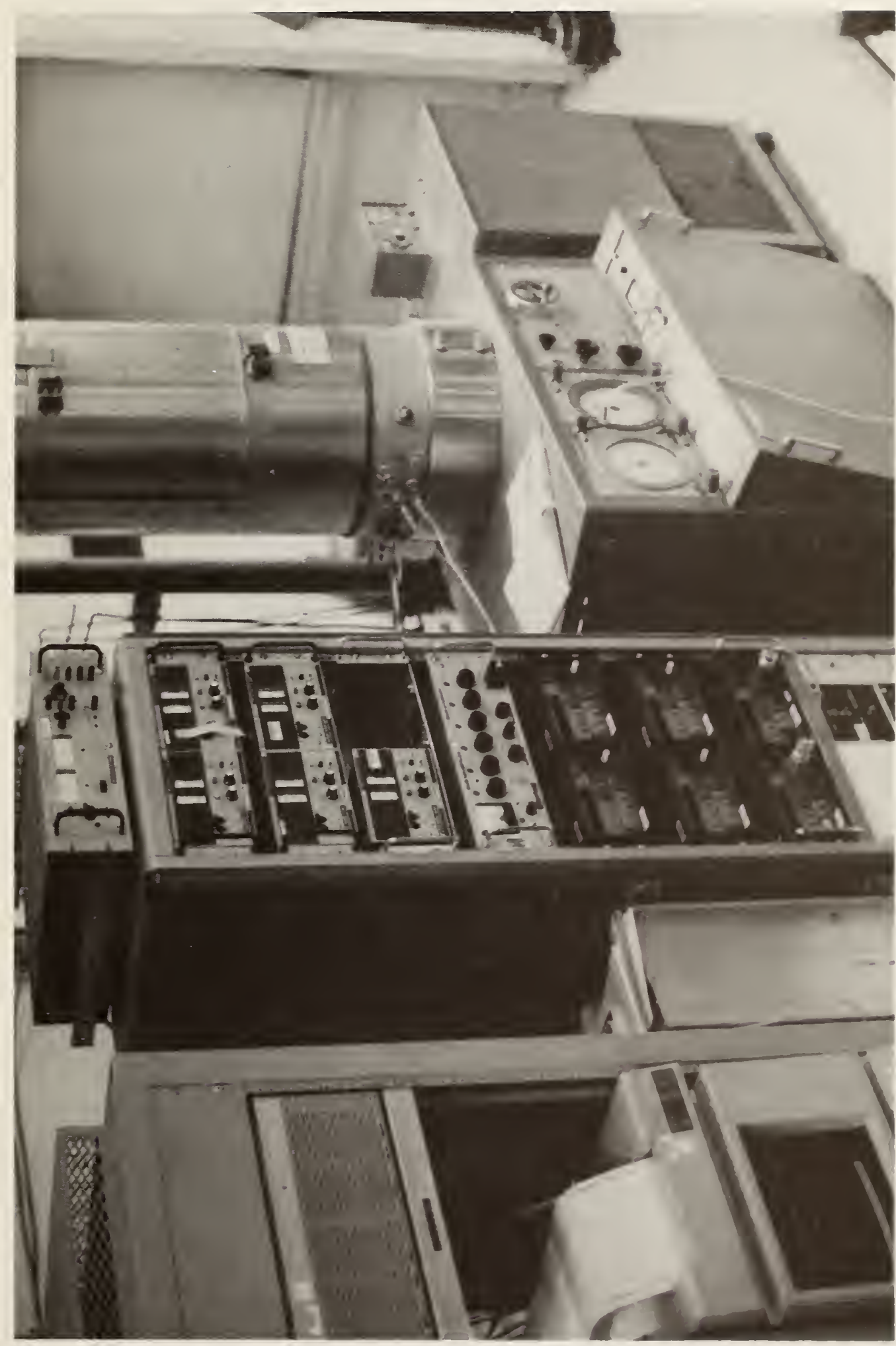




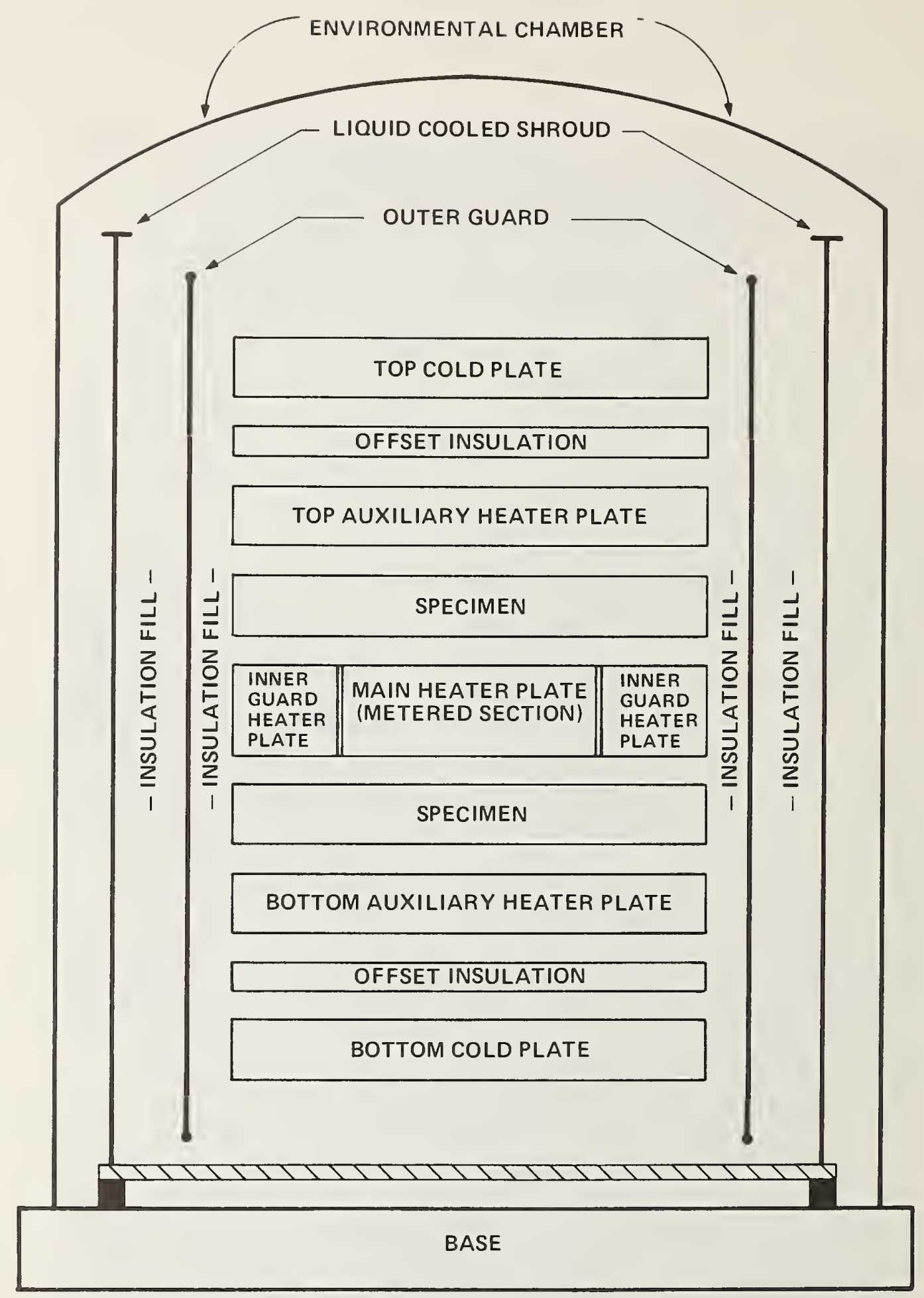

Figure 2. Schematic of guarded-hot-plate specimen configuration and environmental chamber. 


\section{SPECIMENS}

The five specimens studied were supplied by either NASA or a NASA contractor. They consisted of three commercially available polymethacrylimide (PMA) foams, one polyurethane (PU) foam sprayed on an aluminum substrate, and one sandwich composite made up of PMA foam, aluminized polyimide film, glass cloth, and adhesive. The specimens are identified in table 1.

Table 1. Test specimens

SPECIMEN

IDENT IFICATION
PMA 51

PMA 71-2

PMA71-1

COMPOSITE

SOFI

\section{COMMENTS*}

Sandwich construction consisting of: aluminized polyimide film, glass cloth/adhesive, PMA110, adhesive, PMA110, and glass cloth/adhesive Sprayed, fluorocarbon-blown polyurethane foam, measured foam density $=37.6 \mathrm{~kg} / \mathrm{m}^{3}(2.3)$

* The diameter of each specimen was approximately $20.3 \mathrm{~cm}$ ( 8 in).

\section{RESULTS}

The test summary for the entire series is given in table 2. In each case the nominal temperature difference established across the specimen was $20 \mathrm{~K}$. $\dot{A} \mathrm{~s}$ indicated in this table, two specimens were tested in the single-sided TEST MODE. This was necessitated by thickness in the case of COMPOSITE and by having only one SOFI specimen. Single-sided operation involves replacing one of the specimens shown in figure 2 with another, well known material, and conducting the experiments with no temperature gradient across the substitute material.

Table 2. Test summary

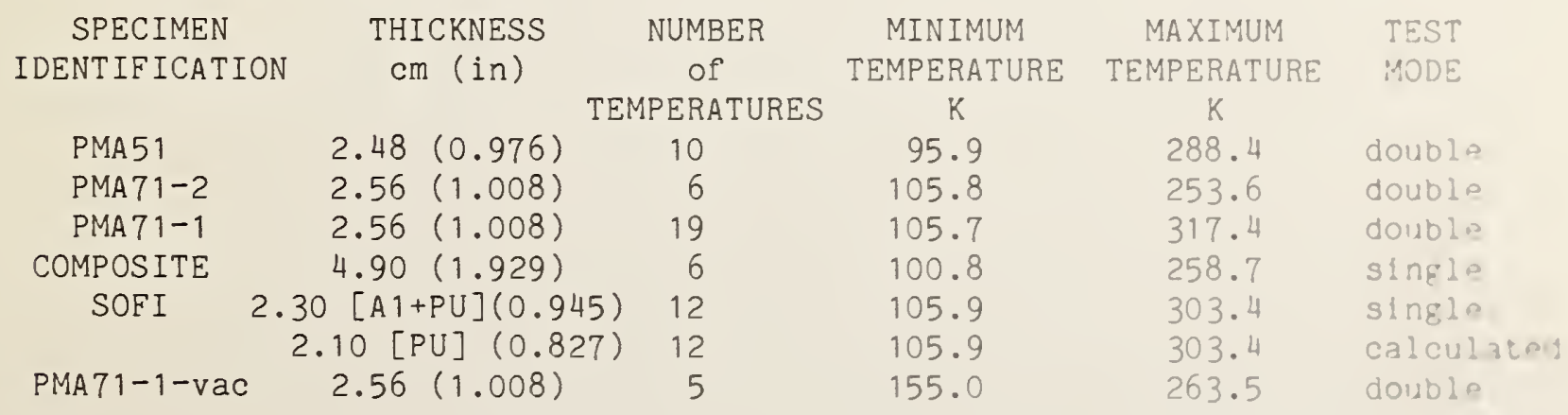


The apparent thermal conductivity, $k$, is computed using eq ( 1 ). The experimentally determined parameters used in this equation are actually averages of the values obtained while the specimen is in thermal equilibrium at a given temperature. For example, once the specimen and the apparatus have reached equilibrium, i.e., constant temperature gradients have been established across the specimen at a constant power input, a series of $\mathrm{N}$ measurements of the power, $Q$, will be made over a period of time, $t$. The average $Q$ and its random uncertainty is computed from these $N$ measurements and is used in eq ( 1 ). The average of the other parameters and their uncertainties are arrived at in a similar manner. These data allow a random uncertainty to be associated with each value of $k$. The values of $k$, computed with eq (1), are referred to as experimental data.

The experimental data for each specimen have been fitted with a power series polynomial of the form

$$
k=\sum_{i=0}^{n} A_{i} T^{i}
$$

The experimental data, polynomial coefficients, tabular values calculated from the fits, plots of $k=f(T)$, and plots of deviations between the fitted and experimental data are presented as indicated in table 3.

Table 3. Location index for all data.

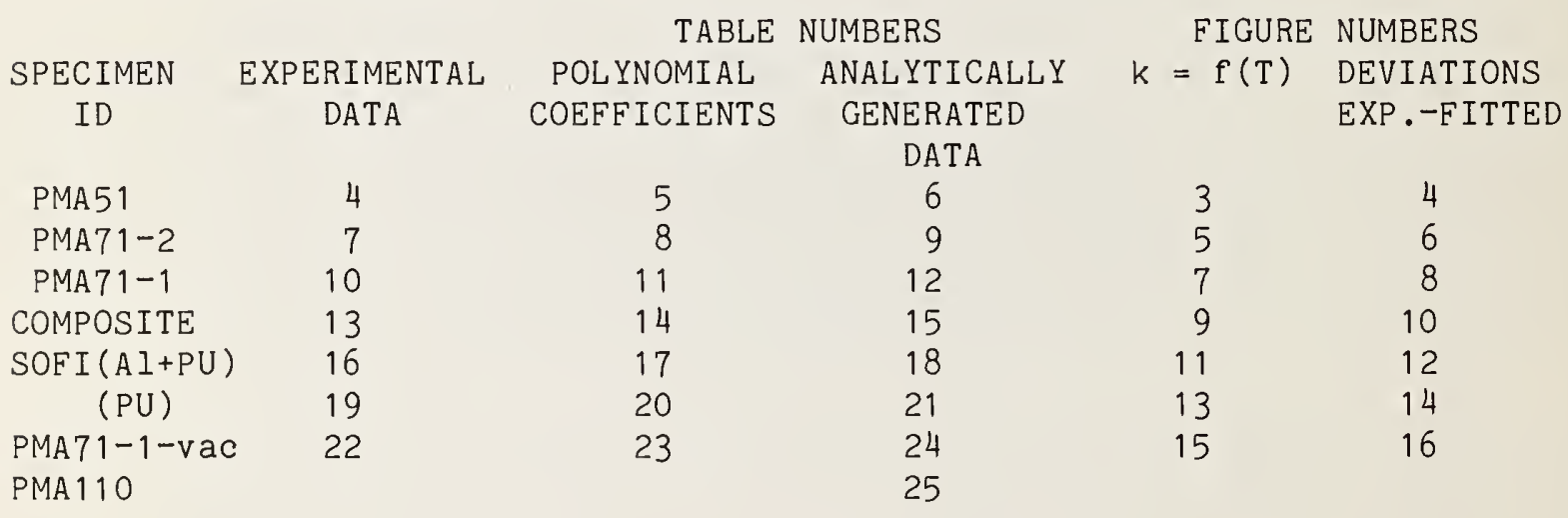


Table 4. Experimental data for PMA51.

TEMPERATURE
$(\mathrm{K})$
228.966
248.657
268.476
288.356
134.959
154.704
174.521
233.904
253.682
95.871

THERMAL CONDUCTIVITY

$(\mathrm{mW} /(\mathrm{m} \cdot \mathrm{K}))$

27.24

29.93

32.72

35.93

17.11

19.14

21.18

28.09

30.86

12.72

Table 5. Polynomial coefficients for a four-term fit of thermal conductivity as a function of temperature for PMA51.

$$
\begin{aligned}
& \mathrm{A} 0=-.13022901 \mathrm{E}+01 \\
& \mathrm{~A} 1=.18710219 \mathrm{E}+00 \\
& \mathrm{~A} 2=-.53477819 \mathrm{E}-03 \\
& \mathrm{~A} 3=.11579740 \mathrm{E}-05
\end{aligned}
$$


Table 6. Thermal conductivity $(\mathrm{mW} /(\mathrm{m} \cdot \mathrm{K}))$ as a function of temperature (K) from the polynomial coefficients given in table 5 for PMA51.

\begin{tabular}{|c|c|c|c|c|c|}
\hline $\mathrm{T}$ & k & $\mathrm{T}$ & k & $T$ & k \\
\hline 96.0 & 12.76 & 162.0 & 19.90 & 226.0 & 27.04 \\
\hline 98.0 & 12.99 & 164.0 & 20.11 & 228.0 & 27.28 \\
\hline 100.0 & 13.22 & 166.0 & $20 \cdot 32$ & 230.0 & 27.53 \\
\hline 102.0 & 13.45 & 168.0 & 20.53 & 232.0 & 27.78 \\
\hline 104.0 & 13.67 & 170.0 & 20.74 & 234.0 & 28.03 \\
\hline 106.0 & 13.90 & 172.0 & 20.95 & 236.0 & 28.29 \\
\hline 108.0 & 14.13 & 174.0 & 21.16 & 238.0 & 28.55 \\
\hline 110.0 & 14.35 & 176.0 & 21.38 & 240.0 & 28.81 \\
\hline 112.0 & 14.57 & 178.0 & 21.59 & 242.0 & 29.07 \\
\hline 114.0 & 14.79 & 180.0 & 21.80 & 244.0 & 29.33 \\
\hline 116.0 & 15.01 & 182.0 & 22.02 & 246.0 & 29.60 \\
\hline 118.0 & 15.23 & 184.0 & 22.23 & 248.0 & 29.87 \\
\hline 120.0 & 15.45 & 186.0 & 22.45 & 250.0 & 30.14 \\
\hline 122.0 & 15.67 & 188.0 & 22.67 & 252.0 & 30.42 \\
\hline 124.0 & 15.88 & 190.0 & 22.88 & 254.0 & 30.70 \\
\hline 126.0 & 16.10 & 192.0 & 23.10 & 256.0 & 30.98 \\
\hline 128.0 & 16.31 & 194.0 & 23.32 & 258.0 & 31.26 \\
\hline 130.0 & 16.53 & 196.0 & 23.54 & 260.0 & 31.55 \\
\hline 132.0 & 16.74 & 198.0 & 23.77 & 262.0 & 31.84 \\
\hline 134.0 & 16.95 & 200.0 & 23.99 & 264.0 & 32.13 \\
\hline 136.0 & 17.17 & 202.0 & 24.22 & 266.0 & 32.42 \\
\hline 138.0 & $17 \cdot 38$ & 204.0 & 24.44 & 268.0 & 32.72 \\
\hline 140.0 & 17.59 & 206.0 & 24.67 & 270.0 & 33.02 \\
\hline 142.0 & 17.80 & 208.0 & 24.90 & 272.0 & 33.33 \\
\hline 144.0 & 18.01 & 210.0 & 25.13 & 274.0 & 33.64 \\
\hline 146.0 & 18.22 & 212.0 & 25.36 & 276.0 & 33.95 \\
\hline 148.0 & 18.43 & 214.0 & 25.60 & 278.0 & 34.26 \\
\hline 150.0 & 18.64 & 216.0 & 25.83 & 280.0 & 34.58 \\
\hline 152.0 & 18.85 & 218.0 & 26.07 & 282.0 & 34.90 \\
\hline 154.0 & 19.06 & 220.0 & 26.31 & 284.0 & 35.23 \\
\hline 156.0 & 19.27 & 222.0 & 26.55 & 286.0 & 35.56 \\
\hline 158.0 & 19.48 & 224.0 & 26.79 & 288.0 & 35.89 \\
\hline 160.0 & 19.69 & & & & \\
\hline
\end{tabular}




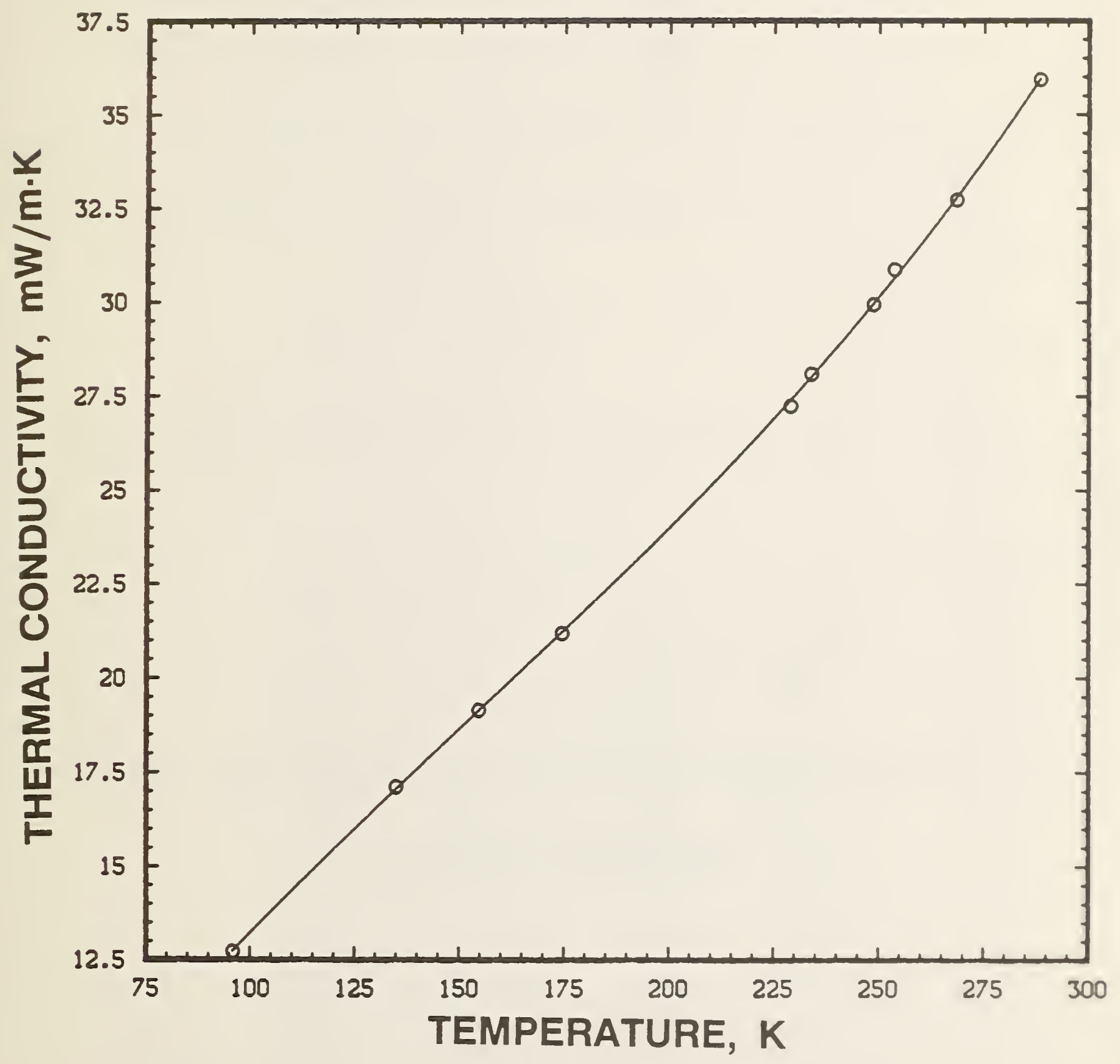

Figure 3. Polynomial fit of thermal conductivity $(\mathrm{mw} /(\mathrm{m} \cdot \mathrm{K})$ ) as a function of temperature (K) for PMA51. Experimental data are represenied by discrete points. 


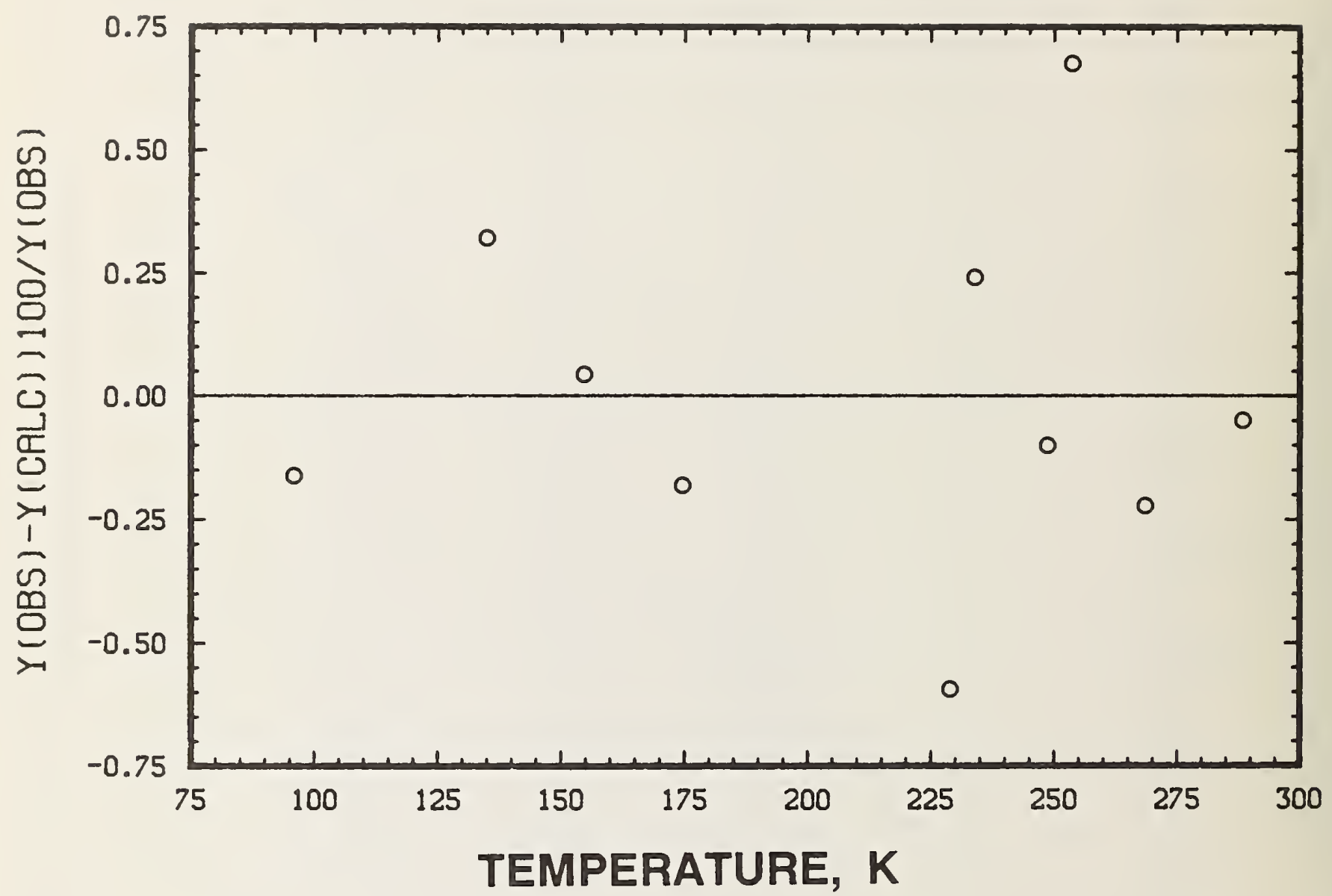

Figure 4. Deviations $(\mathcal{q})$ between experimental and fitted data as a function of temperature (K) for PMA51. 
Table 7. Experimental data for PMA71-2.

$\begin{array}{cc}\text { TEMPERATURE } & \text { THERMAL CONDUCT } \\ (\mathrm{K}) & (\mathrm{mW} /(\mathrm{m} \cdot \mathrm{K})) \\ 229.061 & 28.71 \\ 253.611 & 32.18 \\ 105.769 & 15.02 \\ 130.167 & 17.93 \\ 164.703 & 21.57 \\ 194.235 & 24.80\end{array}$

Table 8. Polynomial coefficients for a four-term fit of thermal conductivity as a function of temperature for PMA71-2.

$$
\begin{aligned}
& \mathrm{A} 0=-.52112427 \mathrm{E}+01 \\
& \mathrm{~A} 1=.27824329 \mathrm{E}+00 \\
& \mathrm{~A} 2=-.10417420 \mathrm{E}-02 \\
& \mathrm{~A} 3=.20725273 \mathrm{E}-05
\end{aligned}
$$

Table 9. Thermal conductivity $(\mathrm{mW} /(\mathrm{m} \cdot \mathrm{K})$ ) as a function of temperature $(\mathrm{K})$ from the polynomial coefficients given in table 8 for PMA71-2.

$\begin{array}{cccccc}T & \mathrm{k} & \mathrm{T} & \mathrm{k} & \mathrm{T} & \mathrm{k} \\ 106.0 & 15.05 & 156.0 & 20.71 & 206.0 & 26.02 \\ 108.0 & 15.30 & 158.0 & 20.92 & 208.0 & 26.24 \\ 110.0 & 15.55 & 160.0 & 21.13 & 210.0 & 26.47 \\ 112.0 & 15.80 & 162.0 & 21.34 & 212.0 & 26.70 \\ 114.0 & 16.04 & 164.0 & 21.54 & 214.0 & 26.94 \\ 116.0 & 16.28 & 166.0 & 21.75 & 216.0 & 27.17 \\ 118.0 & 16.52 & 168.0 & 21.96 & 218.0 & 27.41 \\ 120.0 & 16.76 & 170.0 & 22.17 & 220.0 & 27.65 \\ 122.0 & 16.99 & 172.0 & 22.37 & 222.0 & 27.89 \\ 124.0 & 17.22 & 174.0 & 22.58 & 224.0 & 28.14 \\ 126.0 & 17.45 & 176.0 & 22.79 & 226.0 & 28.39 \\ 128.0 & 17.68 & 178.0 & 23.00 & 228.0 & 28.64 \\ 130.0 & 17.91 & 180.0 & 23.21 & 230.0 & 28.89 \\ 132.0 & 18.13 & 182.0 & 23.42 & 232.0 & 29.15 \\ 134.0 & 18.35 & 184.0 & 23.63 & 234.0 & 29.41 \\ 136.0 & 18.58 & 186.0 & 23.84 & 236.0 & 29.68 \\ 138.0 & 18.79 & 188.0 & 24.05 & 238.0 & 29.94 \\ 140.0 & 19.01 & 190.0 & 24.26 & 240.0 & 30.21 \\ 142.0 & 19.23 & 192.0 & 24.48 & 242.0 & 30.49 \\ 144.0 & 19.44 & 194.0 & 24.69 & 244.0 & 30.77 \\ 146.0 & 19.66 & 196.0 & 24.91 & 246.0 & 31.05 \\ 148.0 & 19.87 & 198.0 & 25.13 & 248.0 & 31.33 \\ 150.0 & 20.08 & 200.0 & 25.35 & 250.0 & 31.62 \\ 152.0 & 20.29 & 202.0 & 25.57 & 252.0 & 31.92 \\ 154.0 & 20.50 & 204.0 & 25.79 & & \end{array}$




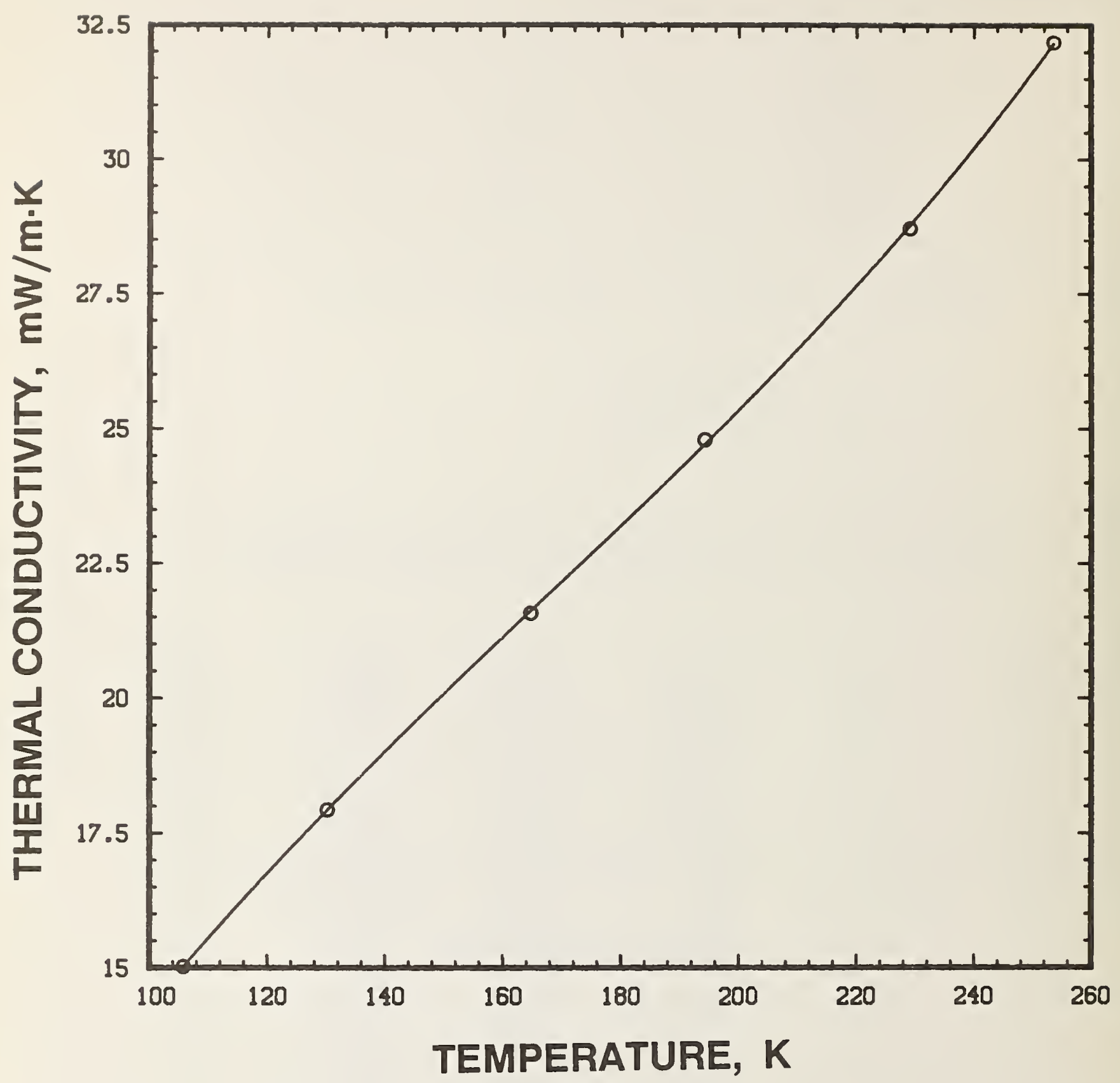

Figure 5. Polynomial fit of thermal conductivity $(\mathrm{mW} /(\mathrm{m} \cdot \mathrm{K}))$ as a function of temperature $(K)$ for PMA71-2. Experimental data are represented by discrete points. 


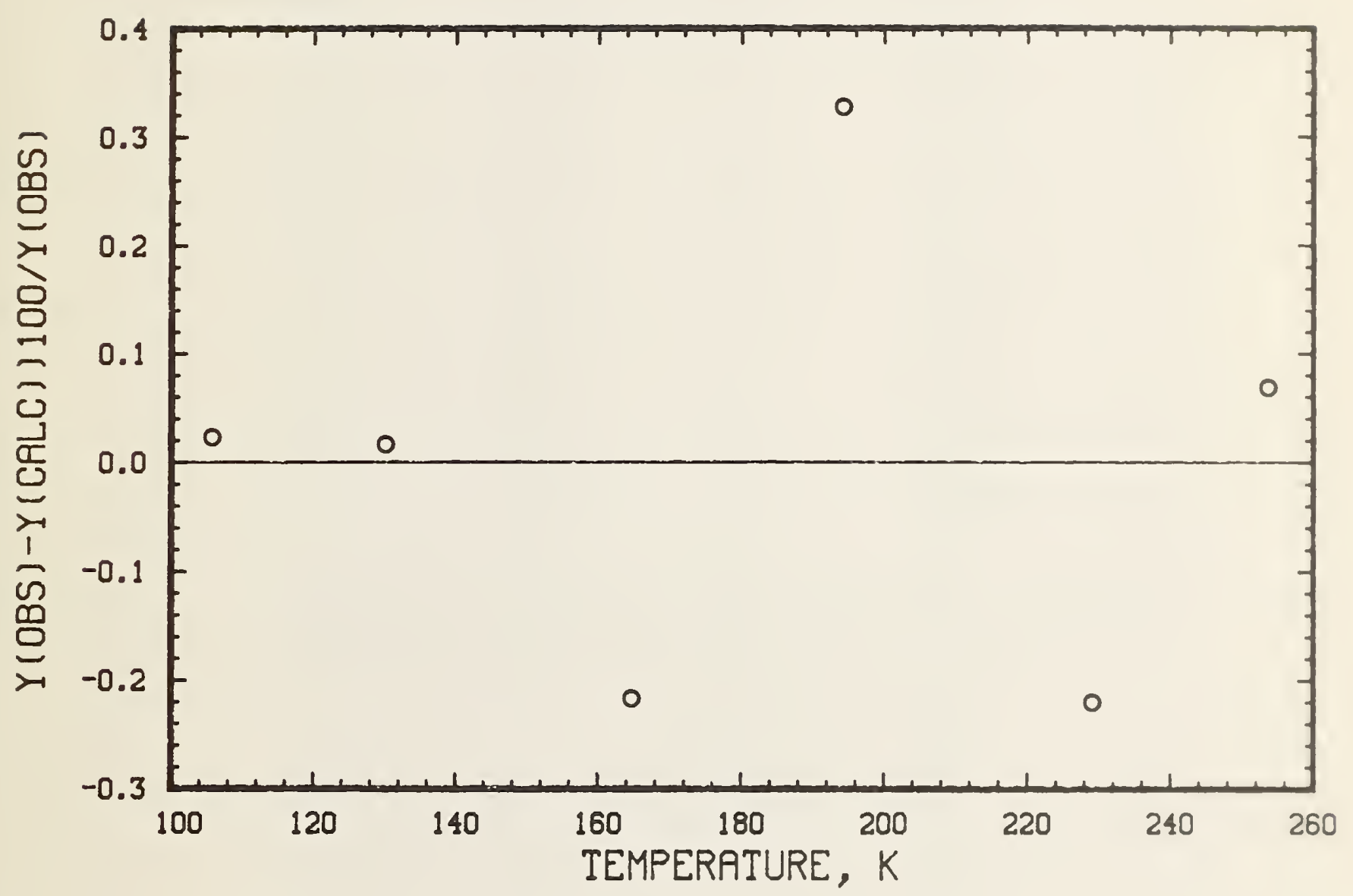

Figure 6 . Deviations $(\%)$ between experimental and fitted data as a function of temperature (K) for PMA71-2. 
Table 10. Experimental data for PMA71-1.

$\begin{array}{cc}\begin{array}{c}\text { TEMPERATURE } \\ (\mathrm{K})\end{array} & \begin{array}{c}\text { THERMAL CONDUCTIVITY } \\ (\mathrm{mW} /(\mathrm{m} \cdot \mathrm{K}))\end{array} \\ 285.390 & 37.28 \\ 251.216 & 31.93 \\ 261.064 & 33.53 \\ 270.901 & 35.25 \\ 317.404 & 43.02 \\ 138.819 & 18.62 \\ 149.494 & 19.73 \\ 159.252 & 20.45 \\ 105.689 & 14.65 \\ 115.326 & 15.82 \\ 125.338 & 16.94 \\ 154.718 & 20.02 \\ 179.663 & 22.51 \\ 204.344 & 25.20 \\ 194.286 & 24.20 \\ 209.119 & 25.82 \\ 224.121 & 27.83 \\ 238.864 & 29.75 \\ 297.795 & 39.97\end{array}$

Table 11. Polynomial coefficients for a five-term fit of thermal conductivity as a function of temperature for PMA71-1.

$$
\begin{aligned}
& \mathrm{AO}=-.24194910 \mathrm{E}+02 \\
& \mathrm{~A} 1=.72667067 \mathrm{E}+00 \\
& \mathrm{~A} 2=-.49017294 \mathrm{E}-02 \\
& \mathrm{~A} 3=.16082458 \mathrm{E}-04 \\
& \mathrm{~A} 4=-.18113899 \mathrm{E}-07
\end{aligned}
$$


Table 12. Thermal conductivity $(\mathrm{mW} /(\mathrm{m} \cdot \mathrm{K})$ ) as a function of temperature (K) from the polynomial coefficients given in table 11 for PiMA1-1.

\begin{tabular}{|c|c|c|c|c|c|}
\hline$T$ & k & $T$ & $k$ & $T$ & k \\
\hline 106.0 & 14.62 & 178.0 & 22.36 & 248.0 & 31.33 \\
\hline 108.0 & 14.91 & 180.0 & 22.57 & 250.0 & 31.65 \\
\hline 110.0 & 15.18 & 182.0 & 22.77 & 252.0 & 31.97 \\
\hline 112.0 & 15.45 & 184.0 & 22.98 & 254.0 & 32.29 \\
\hline 114.0 & 15.71 & 186.0 & 23.19 & 256.0 & 32.61 \\
\hline 116.0 & 15.96 & 188.0 & 23.41 & 258.0 & 32.94 \\
\hline 118.0 & 16.21 & 190.0 & 23.62 & 260.0 & 33.27 \\
\hline 120.0 & 16.46 & 192.0 & 23.84 & 262.0 & 33.60 \\
\hline 122.0 & 16.69 & 194.0 & 24.06 & 264.0 & 33.94 \\
\hline 124.0 & 16.92 & 196.0 & 24.29 & 266.0 & 34.28 \\
\hline 126.0 & 17.15 & 198.0 & 24.52 & 268.0 & 34.62 \\
\hline 128.0 & 17.37 & 200.0 & 24.75 & 270.0 & 34.96 \\
\hline 130.0 & 17.59 & 202.0 & 24.98 & 272.0 & 35.30 \\
\hline 132.0 & 17.81 & 204.0 & 25.22 & 274.0 & 35.64 \\
\hline 134.0 & 18.02 & 206.0 & 25.46 & 276.0 & 35.99 \\
\hline 136.0 & 18.23 & 208.0 & 25.70 & 278.0 & 36.33 \\
\hline 138.0 & 18.43 & 210.0 & 25.95 & 280.0 & 36.68 \\
\hline 140.0 & 18.64 & 212.0 & 26.20 & 282.0 & $37.0=$ \\
\hline 142.0 & 18.84 & 214.0 & 26.46 & 284.0 & 37.38 \\
\hline 144.0 & 19.04 & 216.0 & 26.71 & 286.0 & 37.73 \\
\hline 146.0 & 19.23 & 218.0 & 26.98 & 288.0 & 38.07 \\
\hline 148.0 & 19.43 & 220.0 & 27.24 & 290.0 & 38.42 \\
\hline 150.0 & 19.62 & 222.0 & 27.51 & 292.0 & 38.77 \\
\hline 152.0 & 19.82 & 224.0 & 27.78 & 294.0 & 39.12 \\
\hline 154.0 & 20.01 & 226.0 & 28.06 & 296.0 & 39.4 \\
\hline 156.0 & 20.21 & 228.0 & 28.34 & 298.0 & 39.8 \\
\hline 158.0 & 20.40 & 230.0 & 28.62 & 300.0 & 40.1 \\
\hline 160.0 & 20.59 & 232.0 & 28.91 & 302.0 & 40.5 \\
\hline 162.0 & 20.78 & 234.0 & 29.20 & 304.0 & 40.8 \\
\hline 164.0 & 20.98 & 236.0 & 29.49 & 306.0 & 41.1 \\
\hline 166.0 & 21.17 & 238.0 & 29.79 & 308.0 & 41.5 \\
\hline 168.0 & 21.37 & 240.0 & 30.09 & 310.0 & 41.3 \\
\hline 170.0 & 21.56 & 242.0 & 30.40 & 312.0 & 42.1 \\
\hline 172.0 & 21.76 & 244.0 & 30.70 & 314.0 & 42.5 \\
\hline 174.0 & 21.96 & 246.0 & 31.02 & 316.0 & 42.9 \\
\hline 176.0 & 22.16 & & & & \\
\hline
\end{tabular}




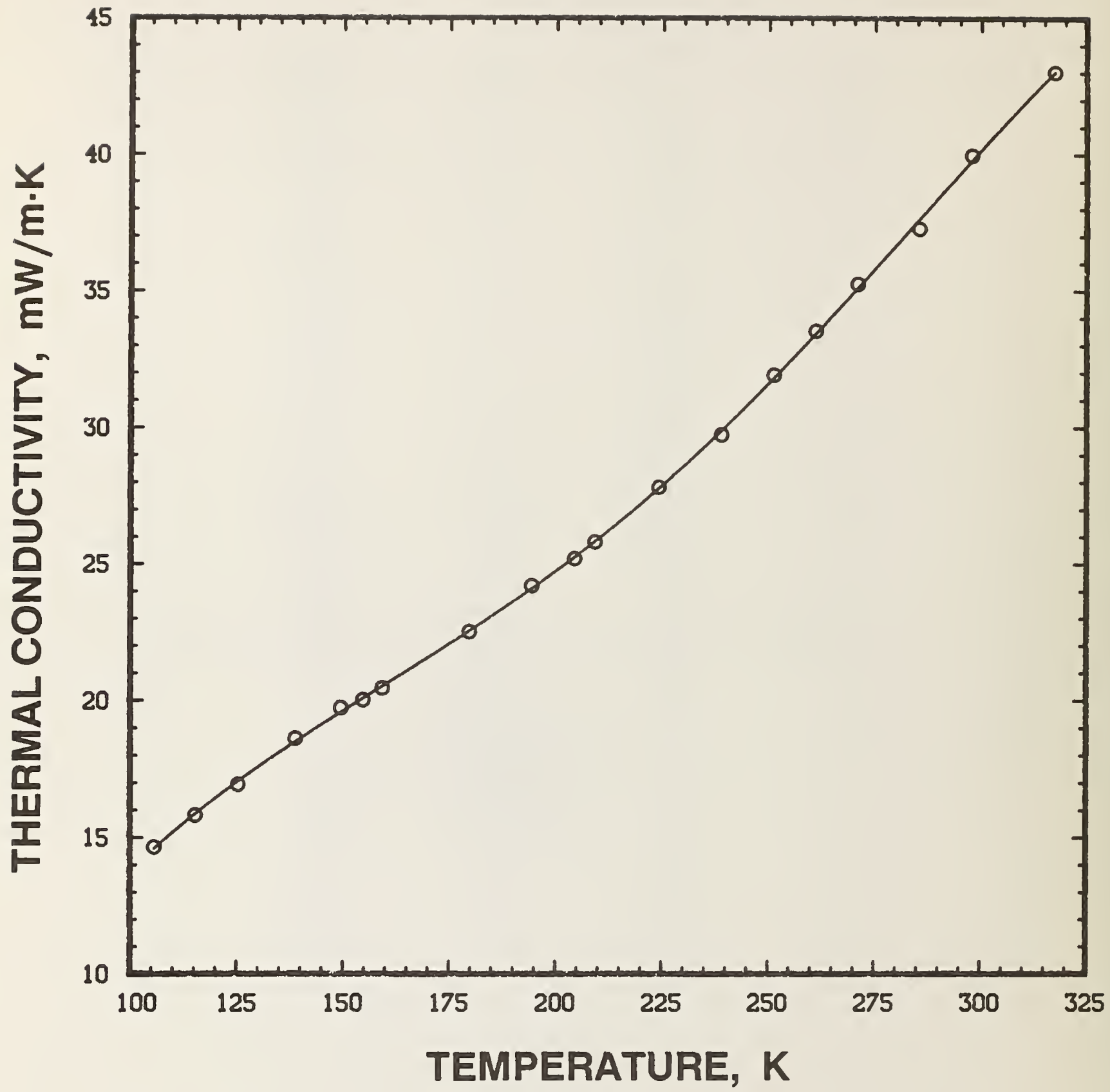

Figure 7. Polynomial fit of thermal conductivity $(\mathrm{mW} /(\mathrm{m} \cdot \mathrm{K}))$ as a function of temperature $(K)$ for PMA71-1. Experimental data are represented by discrete points. 


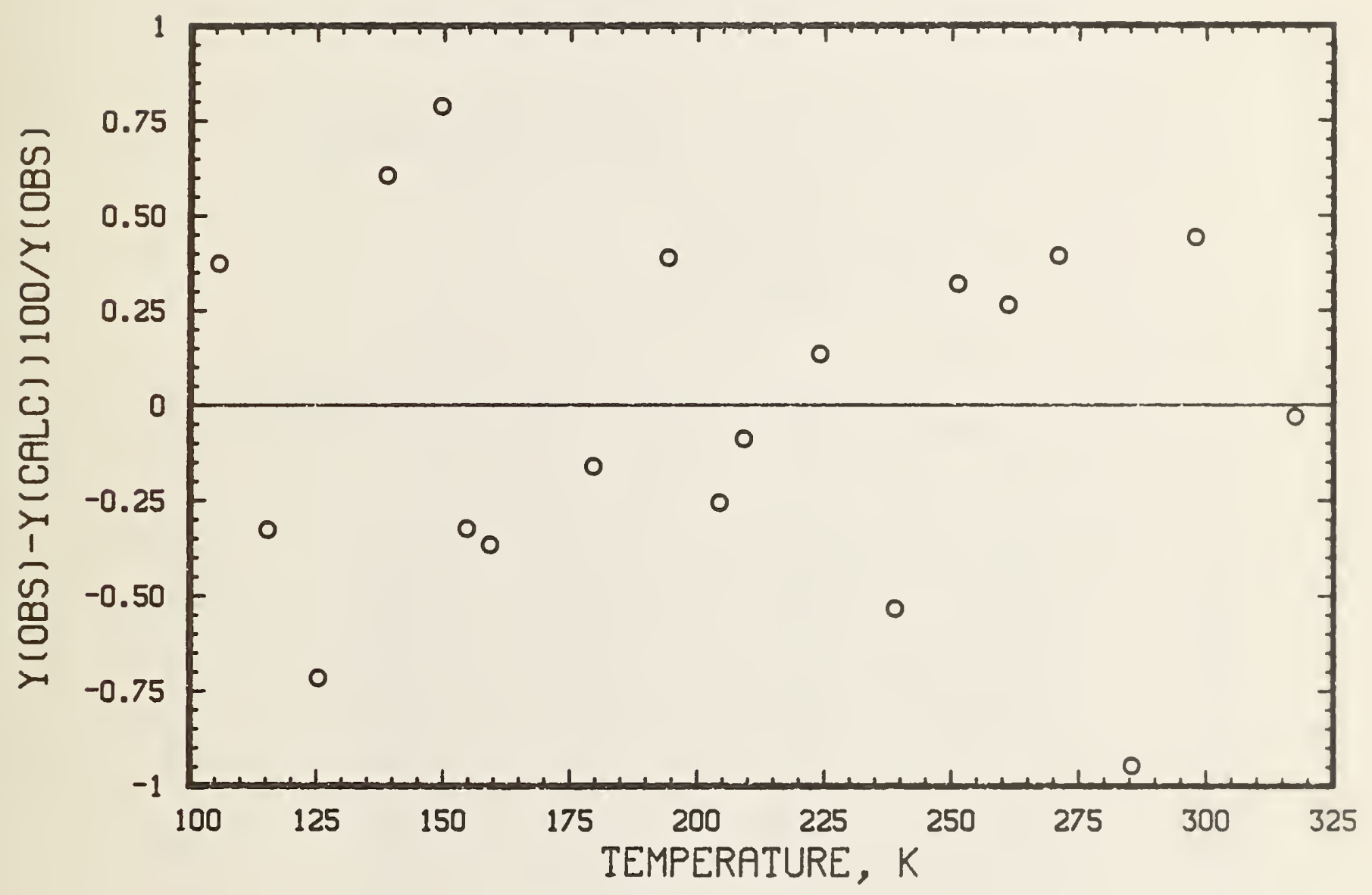

Figure 8. Deviations ( $\%$ ) between experimental and ritted data as a function of temperature (K) for PMA71-1. 
Table 13. Experimental data for COMPOSITE.

$\begin{array}{cc}\begin{array}{c}\text { TEMPERATURE } \\ (\mathrm{K})\end{array} & \begin{array}{r}\text { THERMAL CONDUCTIVITY } \\ (\mathrm{mW} /(\mathrm{m} \cdot \mathrm{K}))\end{array} \\ 233.994 & 33.37 \\ 258.677 & 37.69 \\ 130.102 & 22.24 \\ 159.518 & 25.72 \\ 100.793 & 16.88 \\ 189.179 & 29.27\end{array}$

Table 14. Polynomial coefficients for a four-term fit of thermal conductivity as a function of temperature for COMPOSITE.

$$
\begin{aligned}
& A_{0}=-.27171067 \mathrm{E}+02 \\
& A_{1}=. .70904743 \mathrm{E}+00 \\
& A_{2}=-.32949191 \mathrm{E}-02 \\
& \mathrm{~A}_{3}=. .58809892 \mathrm{E}-05
\end{aligned}
$$

Table 15. Thermal conductivity $(\mathrm{mW} /(\mathrm{m} \cdot \mathrm{K}))$ as a function of temperature $(\mathrm{K})$ from the polynomial coefficients given in table 14 for COMPOSITE.

$\begin{array}{cccccc}\mathrm{T} & \mathrm{k} & \mathrm{T} & \mathrm{k} & \mathrm{T} & \mathrm{k} \\ 100.0 & 16.67 & 154.0 & 25.36 & 208.0 & 30.68 \\ 102.0 & 17.11 & 156.0 & 25.58 & 210.0 & 30.89 \\ 104.0 & 17.55 & 158.0 & 25.80 & 212.0 & 31.09 \\ 106.0 & 17.97 & 160.0 & 26.02 & 214.0 & 31.31 \\ 108.0 & 18.38 & 162.0 & 26.23 & 216.0 & 31.52 \\ 110.0 & 18.78 & 164.0 & 26.43 & 218.0 & 31.74 \\ 112.0 & 19.17 & 166.0 & 26.64 & 220.0 & 31.97 \\ 114.0 & 19.55 & 168.0 & 26.84 & 222.0 & 32.19 \\ 116.0 & 19.92 & 170.0 & 27.04 & 224.0 & 32.43 \\ 118.0 & 20.28 & 172.0 & 27.23 & 226.0 & 32.67 \\ 120.0 & 20.63 & 174.0 & 27.43 & 228.0 & 32.91 \\ 122.0 & 20.97 & 176.0 & 27.62 & 230.0 & 33.16 \\ 124.0 & 21.30 & 178.0 & 27.81 & 232.0 & 33.42 \\ 126.0 & 21.62 & 180.0 & 28.00 & 234.0 & 33.68 \\ 128.0 & 21.94 & 182.0 & 28.19 & 236.0 & 33.95 \\ 130.0 & 22.24 & 184.0 & 28.38 & 238.0 & 34.23 \\ 132.0 & 22.54 & 186.0 & 28.56 & 240.0 & 34.51 \\ 134.0 & 22.83 & 188.0 & 28.75 & 242.0 & 34.80 \\ 136.0 & 23.11 & 190.0 & 28.94 & 244.0 & 35.10 \\ 138.0 & 23.38 & 192.0 & 29.13 & 246.0 & 35.41 \\ 140.0 & 23.65 & 194.0 & 29.32 & 248.0 & 35.72 \\ 142.0 & 23.91 & 196.0 & 29.51 & 250.0 & 36.05 \\ 144.0 & 24.17 & 198.0 & 29.70 & 252.0 & 36.38 \\ 146.0 & 24.42 & 200.0 & 29.89 & 254.0 & 36.72 \\ 148.0 & 24.66 & 202.0 & 30.08 & 256.0 & 37.08 \\ 150.0 & 24.90 & 204.0 & 30.28 & 258.0 & 37.44 \\ 152.0 & 25.13 & 206.0 & 30.48 & & \end{array}$




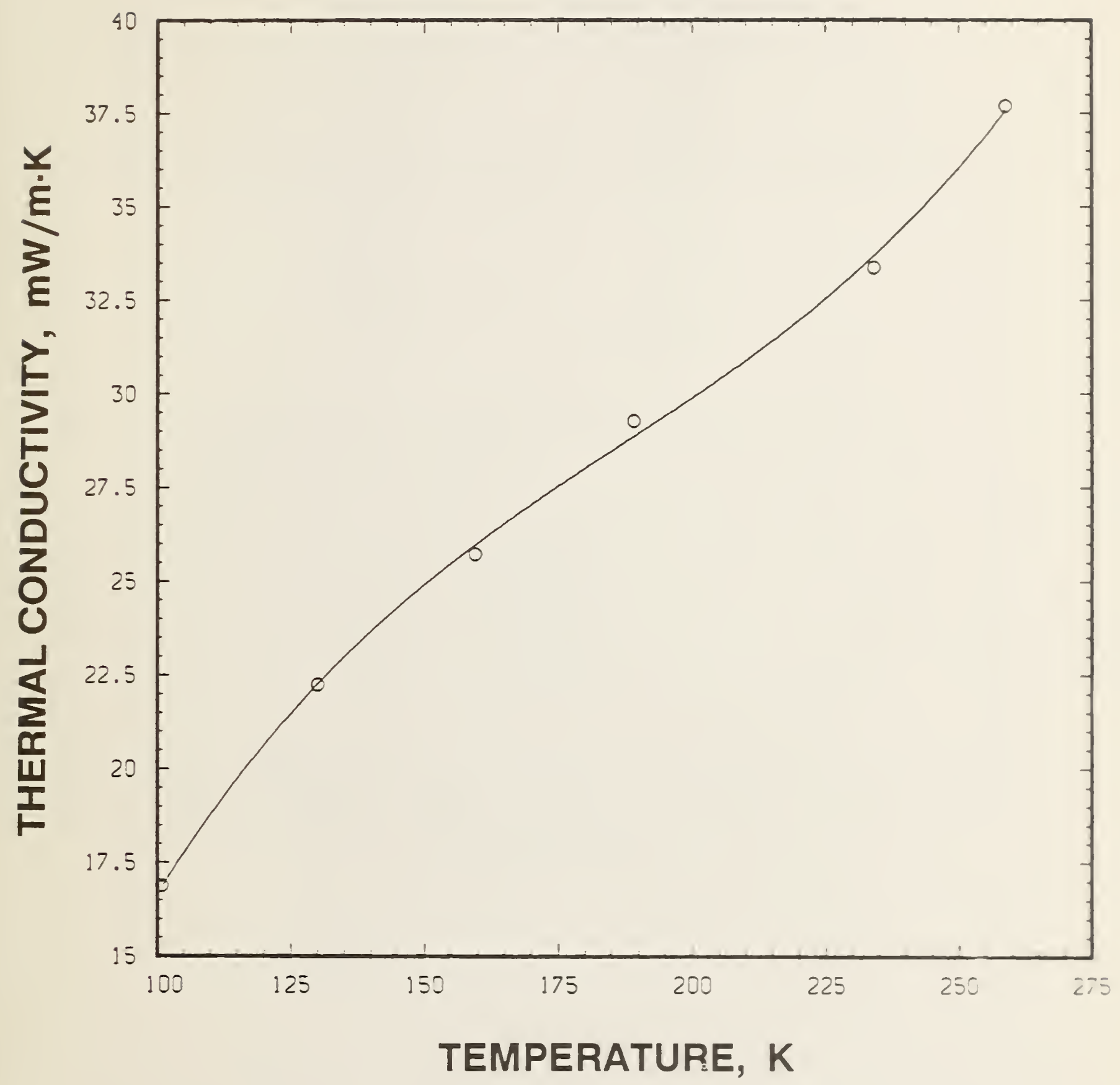

Figure 9. Polynomial fit of thermal conductivity $(\mathrm{min} /(\mathrm{m} \cdot \mathrm{K}))$ as a function of temperature (K) for COMPOSITE. Experimental data are represented by discrete points. 


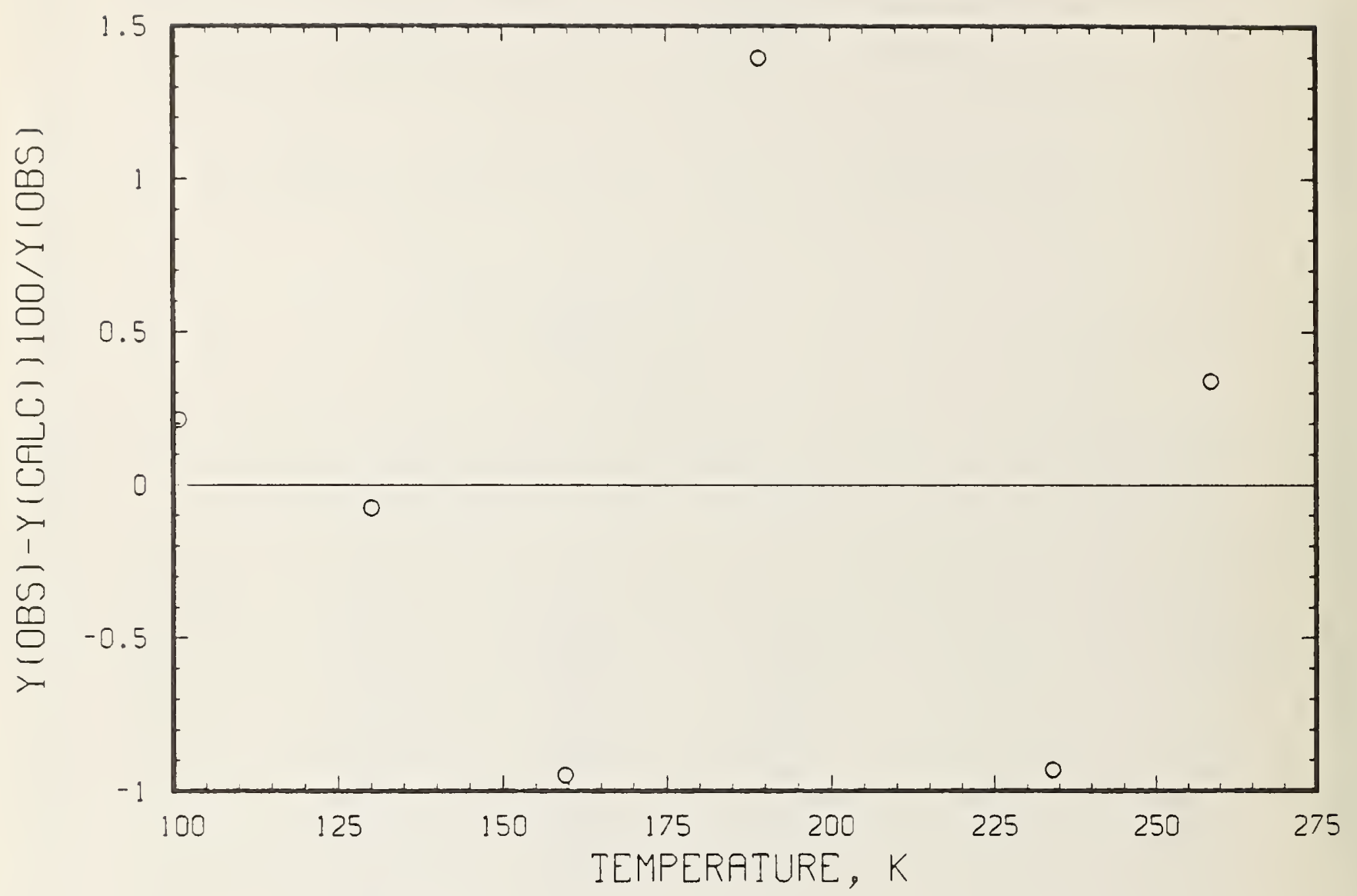

Figure 10. Deviations $(\%)$ between experimental and fitted data as a function of temperature $(K)$ for COMPOSITE. 
Table 16. Experimental data for an aluminum/foam composite specimen, SOFI.

$\begin{array}{cc}\begin{array}{c}\text { TEMPERATURE } \\ (\mathrm{K})\end{array} & \begin{array}{c}\text { THERMAL CONDUCTIVITY } \\ (\mathrm{mW} /(\mathrm{m} \cdot \mathrm{K}))\end{array} \\ 105.788 & 15.00 \\ 125.255 & 17.60 \\ 144.932 & 20.52 \\ 164.538 & 23.01 \\ 184.286 & 25.43 \\ 204.193 & 27.26 \\ 233.973 & 26.68 \\ 224.085 & 27.22 \\ 243.750 & 25.71 \\ 263.635 & 24.53 \\ 283.488 & 26.30 \\ 303.443 & 28.12\end{array}$

Table 17. Polynomial coefficients for a seven-term fit of thermal conductivity as a function of temperature for an aluminum/ foam composite, SOFI.

$$
\begin{aligned}
& \mathrm{AO}=-.74495171 \mathrm{E}+03 \\
& \mathrm{~A} 1=.25852200 \mathrm{E}+02 \\
& \mathrm{~A} 2=-.35813007 \mathrm{E}+00 \\
& \mathrm{~A} 3=.25738209 \mathrm{E}-02 \\
& \mathrm{~A} 4=-.10054371 \mathrm{E}-04 \\
& \mathrm{~A} 5=.20228815 \mathrm{E}-07 \\
& \mathrm{~A} 6=-.16405909 \mathrm{E}-10
\end{aligned}
$$


Table 18. Thermal conductivity $(\mathrm{mW} /(\mathrm{m} \cdot \mathrm{K})$ ) as a function of temperature $(\mathrm{K})$ from the polynomial coefficients given in table 17 for an aluminum/foam composite, SOFI.

\begin{tabular}{|c|c|c|c|c|c|}
\hline $\mathrm{T}$ & k & $\mathrm{T}$ & k & $\mathrm{T}$ & $\mathrm{k}$ \\
\hline 106.0 & 14.99 & 172.0 & 24.14 & 238.0 & 26.16 \\
\hline 108.0 & 15.44 & 174.0 & 24.43 & 240.0 & 26.02 \\
\hline 110.0 & 15.83 & 176.0 & 24.71 & 242.0 & 25.89 \\
\hline 112.0 & 16.19 & 178.0 & 24.98 & 244.0 & 25.75 \\
\hline 114.0 & 16.50 & 180.0 & 25.25 & 246.0 & 25.62 \\
\hline 116.0 & 16.79 & 182.0 & 25.50 & 248.0 & 25.50 \\
\hline 118.0 & 17.05 & 184.0 & 25.74 & 250.0 & 25.39 \\
\hline 120.0 & 17.30 & 186.0 & 25.97 & 252.0 & 25.29 \\
\hline 122.0 & 17.53 & 188.0 & 26.18 & 254.0 & 25.19 \\
\hline 124.0 & 17.75 & 190.0 & 26.38 & 256.0 & 25.12 \\
\hline 126.0 & 17.96 & 192.0 & 26.56 & 258.0 & 25.06 \\
\hline 128.0 & 18.17 & 194.0 & 26.72 & 260.0 & 25.02 \\
\hline 130.0 & 18.39 & 196.0 & 26.87 & 262.0 & 24.98 \\
\hline 132.0 & 18.60 & 198.0 & 26.99 & 264.0 & 24.98 \\
\hline 134.0 & 18.82 & 200.0 & 27.10 & 266.0 & 24.99 \\
\hline 136.0 & 19.04 & 202.0 & 27.19 & 268.0 & 25.03 \\
\hline 138.0 & 19.27 & 204.0 & 27.26 & 270.0 & 25.08 \\
\hline 140.0 & 19.51 & 206.0 & $27 \cdot 32$ & 272.0 & 25.16 \\
\hline 142.0 & 19.76 & 208.0 & 27.35 & 274.0 & 25.26 \\
\hline 144.0 & 20.01 & 210.0 & $27 \cdot 37$ & 276.0 & 25.38 \\
\hline 146.0 & 20.27 & 212.0 & 27.37 & 278.0 & 25.51 \\
\hline 148.0 & 20.55 & 214.0 & 27.35 & 280.0 & 25.68 \\
\hline 150.0 & 20.82 & 216.0 & $27 \cdot 32$ & 282.0 & 25.85 \\
\hline 152.0 & 21.11 & 218.0 & 27.27 & 284.0 & 26.05 \\
\hline 154.0 & 21.40 & 220.0 & 27.20 & 286.0 & 26.26 \\
\hline 156.0 & 21.70 & 222.0 & 27.12 & 288.0 & 26.48 \\
\hline 158.0 & 22.00 & 224.0 & 27.03 & 290.0 & 26.72 \\
\hline 160.0 & 22.31 & 226.0 & 26.93 & 292.0 & 26.96 \\
\hline 162.0 & 22.62 & 228.0 & 26.82 & 294.0 & 27.19 \\
\hline 164.0 & 22.92 & 230.0 & 26.70 & 296.0 & 27.43 \\
\hline 166.0 & 23.23 & 232.0 & 26.57 & 298.0 & 27.65 \\
\hline 168.0 & 23.54 & 234.0 & 26.44 & 300.0 & 27.87 \\
\hline 170.0 & 23.84 & 236.0 & 26.30 & 302.0 & 28.07 \\
\hline
\end{tabular}




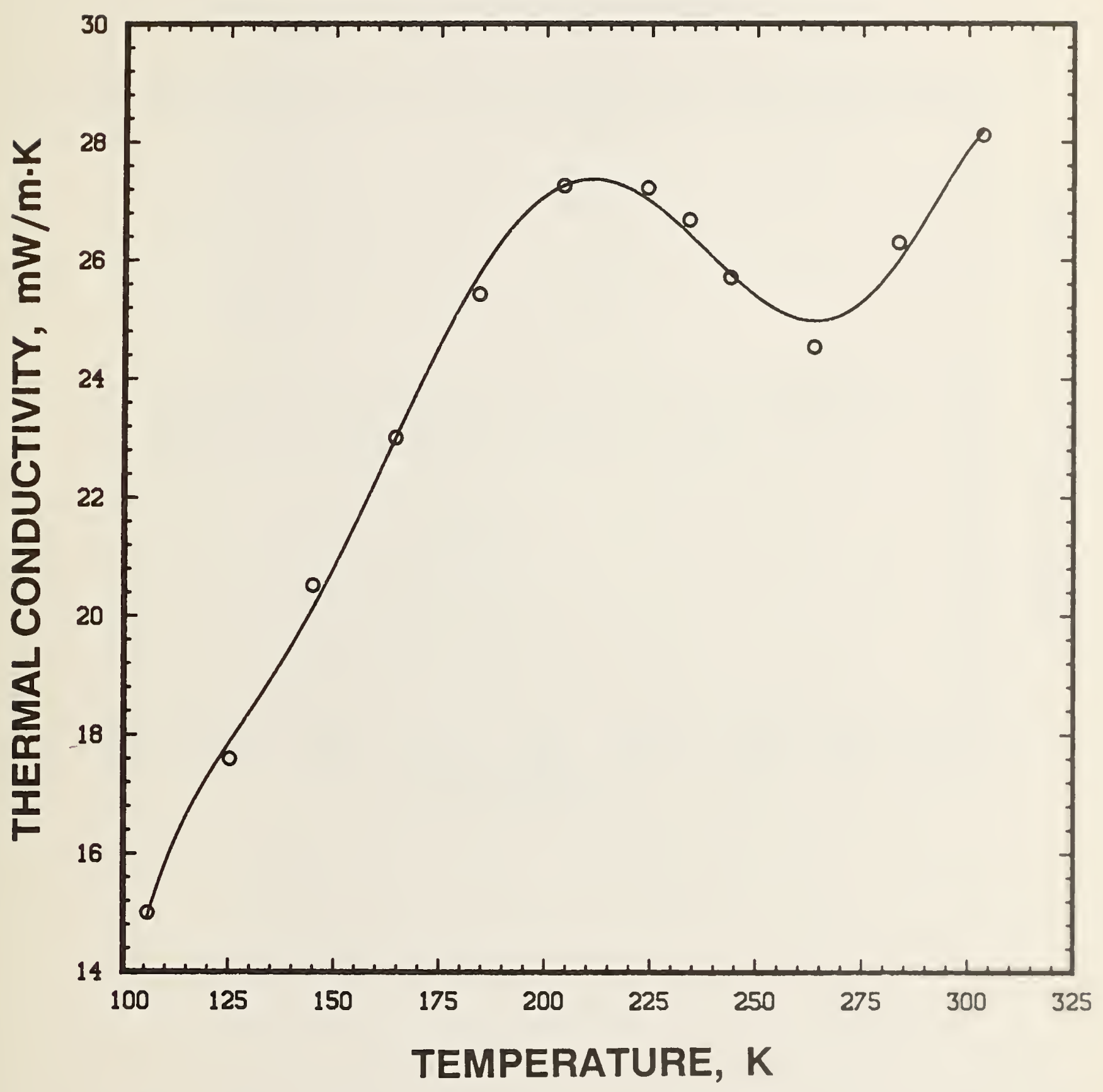

Figure 11. Polynomial fit of thermal conductivity $(\mathrm{mW} /(\mathrm{m} \cdot \mathrm{K}))$ as a function of temperature (K) for an aluminum/foam composite. SOFI. Experimental data are represented by discrete polnts. 


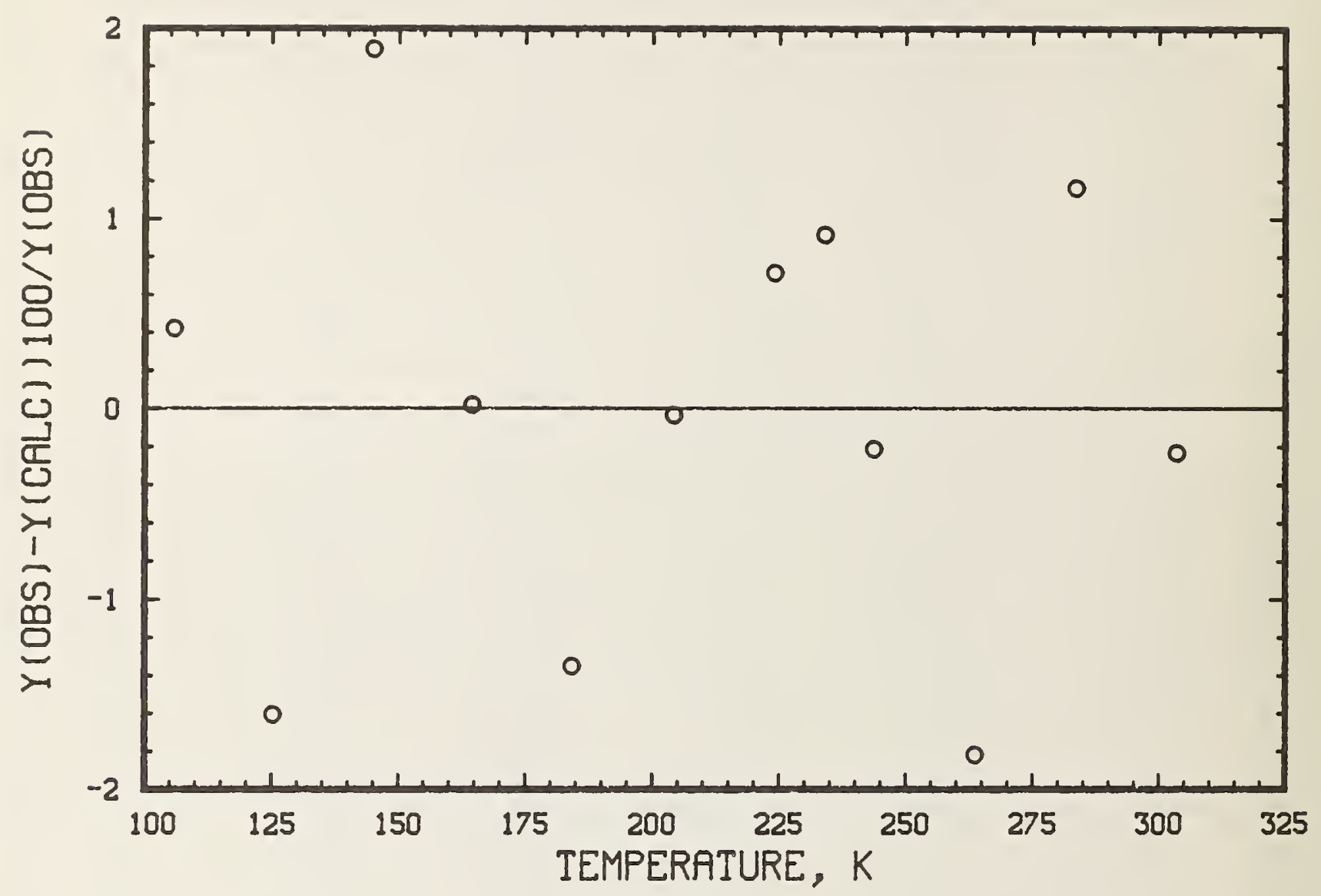

Figure 12. Deviations $(\%)$ between experimental and fitted data as a function of temperature (K) for an aluminum/foam composite, SOFI. 
Table 19. Computed thermal conductivity of the foam component of an aluminum/foam composite, SOFI.

$\begin{array}{cc}\begin{array}{c}\text { TEMPERATURE } \\ (\mathrm{K})\end{array} & \begin{array}{c}\text { THERMA CONDUCTIVITY } \\ (\mathrm{mW} /(\mathrm{m} \cdot \mathrm{K}))\end{array} \\ 105.789 & 13.11 \\ 125.256 & 15.37 \\ 144.932 & 17.90 \\ 164.538 & 20.09 \\ 184.286 & 22.18 \\ 204.193 & 23.75 \\ 233.974 & 23.34 \\ 224.085 & 23.80 \\ 243.751 & 22.52 \\ 263.636 & 21.52 \\ 283.488 & 22.97 \\ 303.443 & 24.55\end{array}$

Table 20. Polynomial coefficients for a seven-term fit of thermal conductivity as a function of temperature for the foam component of an aluminum/foam composite, SOFI.

$$
\begin{aligned}
& \mathrm{AO}=-.61108028 \mathrm{E}+03 \\
& \mathrm{~A} 1=.21218929 \mathrm{E}+02 \\
& \mathrm{~A} 2=-.29384951 \mathrm{E}+00 \\
& \mathrm{~A} 3=.21113743 \mathrm{E}-02 \\
& \mathrm{~A} 4=-.82434748 \mathrm{E}-05 \\
& \mathrm{~A} 5=.16570060 \mathrm{E}-07 \\
& \mathrm{~A} 6=-.13421171 \mathrm{E}-10
\end{aligned}
$$


Table 21. Thermal conductivity $(\mathrm{mW} /(\mathrm{m} \cdot \mathrm{K}))$ as a function of temperature $(\mathrm{K})$ from the coefficients given in table 20 for the foam component of an aluminum/foam composite, SOFI.

$\begin{array}{cccccc}T & \mathrm{~T} & \mathrm{~T} & \mathrm{~T} & \mathrm{k} \\ 106.0 & 13.10 & 172.0 & 21.05 & 238.0 & 22.91 \\ 108.0 & 13.48 & 174.0 & 21.30 & 240.0 & 22.79 \\ 110.0 & 13.82 & 176.0 & 21.55 & 242.0 & 22.68 \\ 112.0 & 14.12 & 178.0 & 21.78 & 244.0 & 22.57 \\ 114.0 & 14.40 & 180.0 & 22.01 & 246.0 & 22.46 \\ 116.0 & 14.65 & 182.0 & 22.23 & 248.0 & 22.36 \\ 118.0 & 14.87 & 184.0 & 22.44 & 250.0 & 22.26 \\ 120.0 & 15.09 & 186.0 & 22.63 & 252.0 & 22.17 \\ 122.0 & 15.29 & 188.0 & 22.82 & 254.0 & 22.09 \\ 124.0 & 15.48 & 190.0 & 22.99 & 256.0 & 22.03 \\ 126.0 & 15.67 & 192.0 & 23.15 & 258.0 & 21.97 \\ 128.0 & 15.86 & 194.0 & 23.29 & 260.0 & 21.94 \\ 130.0 & 16.05 & 196.0 & 23.42 & 262.0 & 21.91 \\ 132.0 & 16.24 & 198.0 & 23.53 & 264.0 & 21.90 \\ 134.0 & 16.43 & 200.0 & 23.63 & 266.0 & 21.90 \\ 136.0 & 16.63 & 202.0 & 23.71 & 268.0 & 21.93 \\ 138.0 & 16.83 & 204.0 & 23.77 & 270.0 & 21.97 \\ 140.0 & 17.04 & 206.0 & 23.82 & 272.0 & 22.03 \\ 142.0 & 17.26 & 208.0 & 23.86 & 274.0 & 22.11 \\ 144.0 & 17.48 & 210.0 & 23.88 & 276.0 & 22.21 \\ 146.0 & 17.71 & 212.0 & 23.88 & 278.0 & 22.32 \\ 148.0 & 17.95 & 214.0 & 23.87 & 280.0 & 22.45 \\ 150.0 & 18.19 & 216.0 & 23.85 & 282.0 & 22.60 \\ 152.0 & 18.44 & 218.0 & 23.81 & 284.0 & 22.76 \\ 154.0 & 18.69 & 220.0 & 23.76 & 286.0 & 22.94 \\ 156.0 & 18.95 & 222.0 & 23.70 & 288.0 & 23.13 \\ 158.0 & 19.21 & 224.0 & 23.62 & 290.0 & 23.32 \\ 160.0 & 19.47 & 226.0 & 23.54 & 292.0 & 23.53 \\ 162.0 & 19.74 & 228.0 & 23.45 & 294.0 & 23.73 \\ 164.0 & 20.01 & 230.0 & 23.35 & 296.0 & 23.94 \\ 166.0 & 20.27 & 232.0 & 23.24 & 298.0 & 24.13 \\ 168.0 & 20.53 & 234.0 & 23.14 & 300.0 & 24.33 \\ 170.0 & 20.79 & 236.0 & 23.02 & 302.0 & 24.50\end{array}$




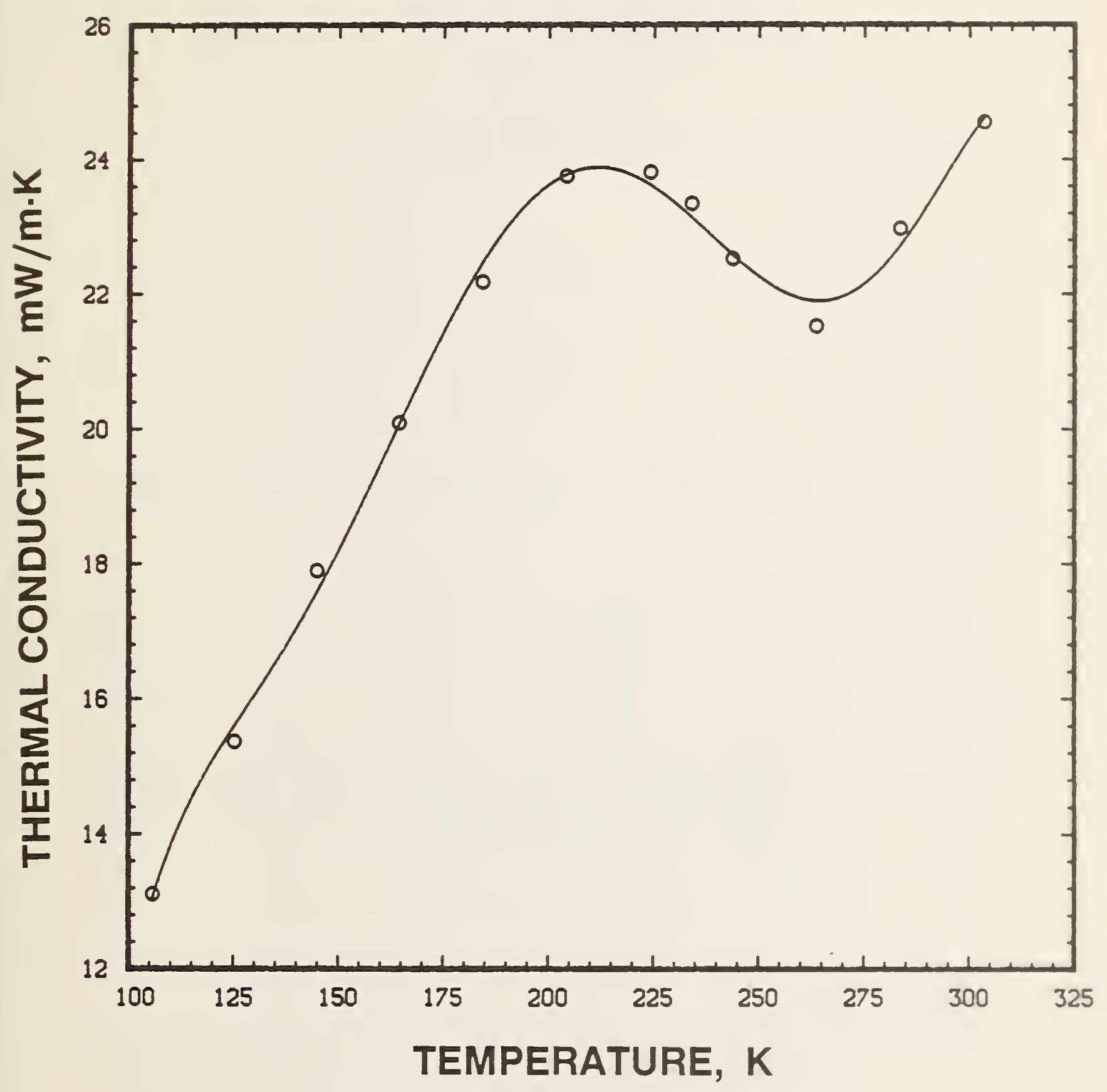

Figure 13. Polynomial fit of thermal conductivity $(\mathrm{mW} /(\mathrm{m} \cdot \mathrm{K})$ ) as a function of temperature $(K)$ for the foam component of an aluminum/foam composite, SOFI. Experimental data are represented by discrete points. 


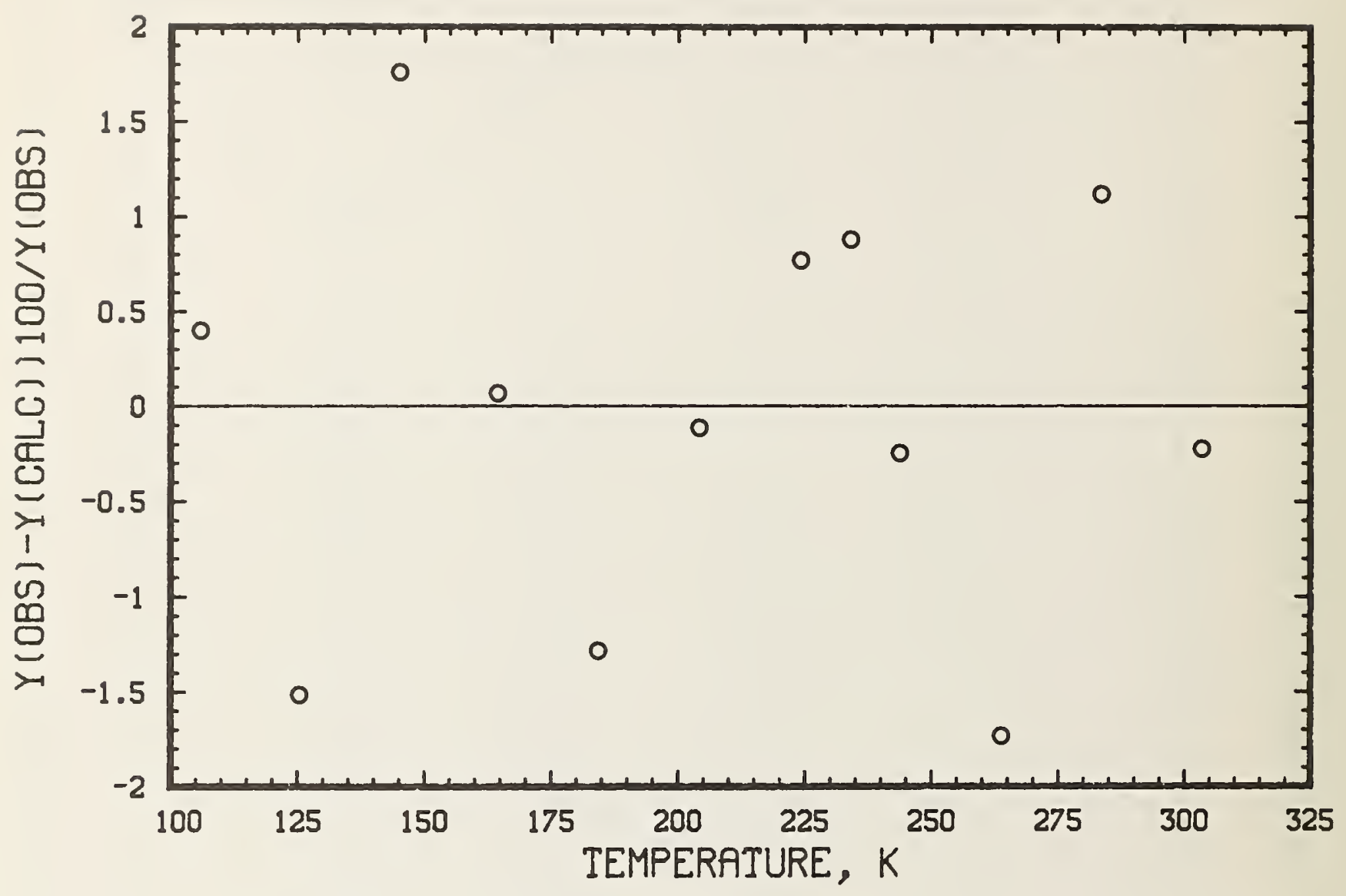

Figure 14. Deviations $(\%)$ between experimental and fitted data as a function of temperature (K) for the foam component of an aluminum/foam composite, SOFI. 
Table 22. Experimental data for PMA71-1-vac.

$\begin{array}{cc}\text { TEMPERATURE } & \text { THERMAL CONDUCTIVITY } \\ (\mathrm{K}) & (\mathrm{mW} /(\mathrm{m} \cdot \mathrm{K})) \\ 263.525 & 33.32 \\ 263.455 & 25.04 \\ 243.695 & 20.48 \\ 223.960 & 17.09 \\ 184.457 & 11.97 \\ 154.989 & 9.56\end{array}$

Table 23. Polynomial coefficients for a three-term fit of thermal conductivity as a function of temperature for PMA71-i-vac.

$$
\begin{aligned}
& A 0=.21870026 E+02 \\
& A 1=-.20846164 E+00 \\
& A 2=.83527367 E-03
\end{aligned}
$$

Table 24. Thermal conductivity $(\mathrm{mW} /(\mathrm{m} \cdot \mathrm{K})$ ) as a function of temperature (K) from the polynomial coefficients given in table 23 for PMA71-1-vac.

$\begin{array}{cccccc}T & k & T & k & T & k \\ 156.0 & 9.68 & 192.0 & 12.64 & 228.0 & 17.75 \\ 158.0 & 9.78 & 194.0 & 12.86 & 230.0 & 18.11 \\ 160.0 & 9.90 & 196.0 & 13.10 & 232.0 & 18.45 \\ 162.0 & 10.02 & 198.0 & 13.34 & 234.0 & 18.83 \\ 164.0 & 10.15 & 200.0 & 13.59 & 236.0 & 19.19 \\ 166.0 & 10.28 & 202.0 & 13.84 & 238.0 & 19.57 \\ 168.0 & 10.42 & 204.0 & 14.10 & 240.0 & 19.95 \\ 170.0 & 10.57 & 206.0 & 14.37 & 242.0 & 20.34 \\ 172.0 & 10.73 & 208.0 & 14.65 & 244.0 & 20.73 \\ 174.0 & 10.89 & 210.0 & 14.93 & 246.0 & 21.14 \\ 176.0 & 11.05 & 212.0 & 15.22 & 248.0 & 21.54 \\ 178.0 & 11.23 & 214.0 & 15.51 & 250.0 & 21.96 \\ 180.0 & 11.41 & 216.0 & 15.81 & 252.0 & 22.38 \\ 182.0 & 11.60 & 218.0 & 16.12 & 254.0 & 22.81 \\ 184.0 & 11.79 & 220.0 & 16.44 & 256.0 & 23.24 \\ 186.0 & 11.99 & 222.0 & 16.76 & 258.0 & 23.69 \\ 188.0 & 12.20 & 224.0 & 17.09 & 260.0 & 24.13 \\ 190.0 & 12.42 & 226.0 & 17.42 & 262.0 & 24.59\end{array}$




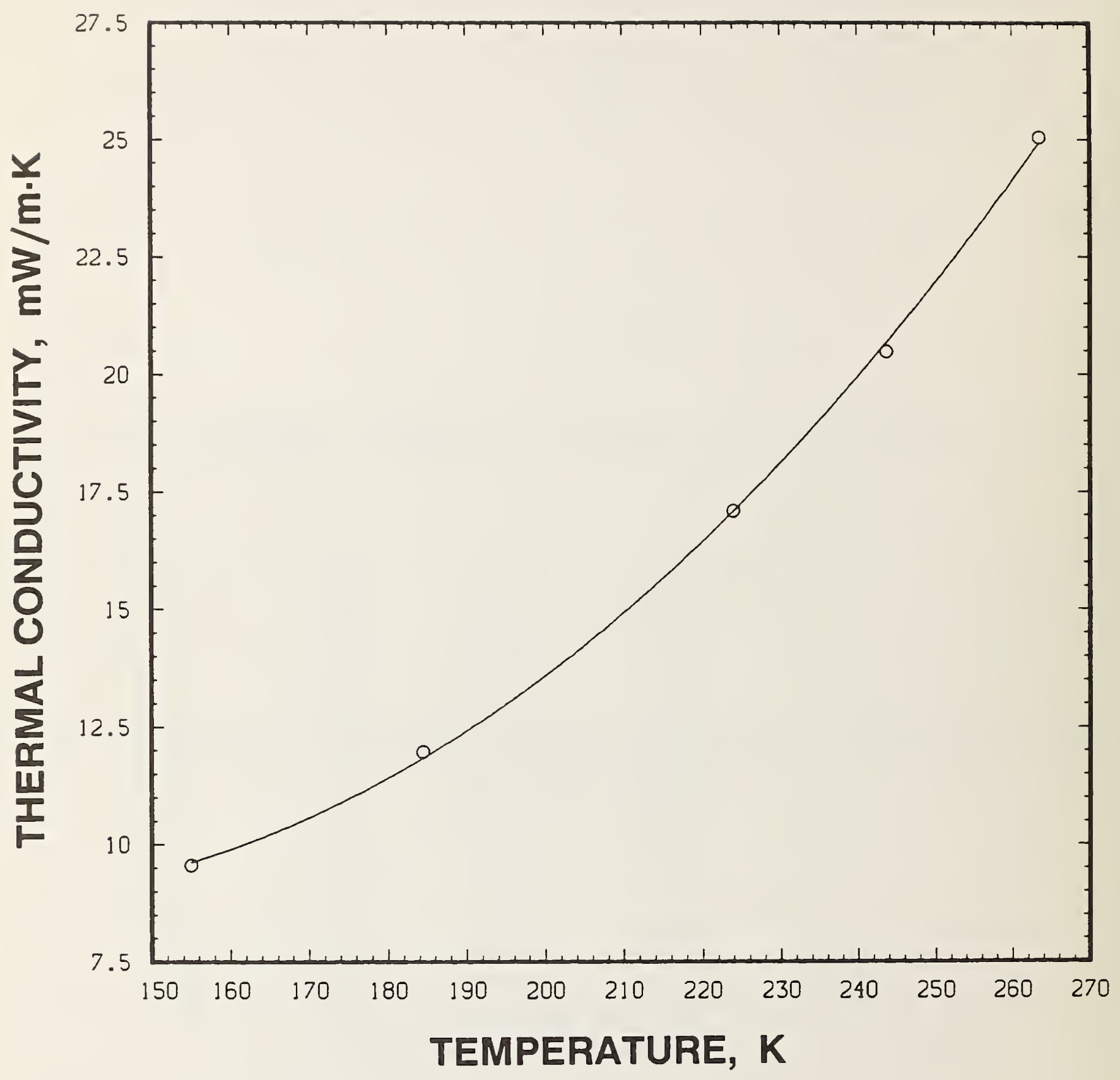

Figure 15. Polynomial fit of thermal conductivity $(\mathrm{mW} /(\mathrm{m} \cdot \mathrm{K}))$ as a function of temperature (K) for PMA71-1-vac. Experimental data are represented by discrete points. 


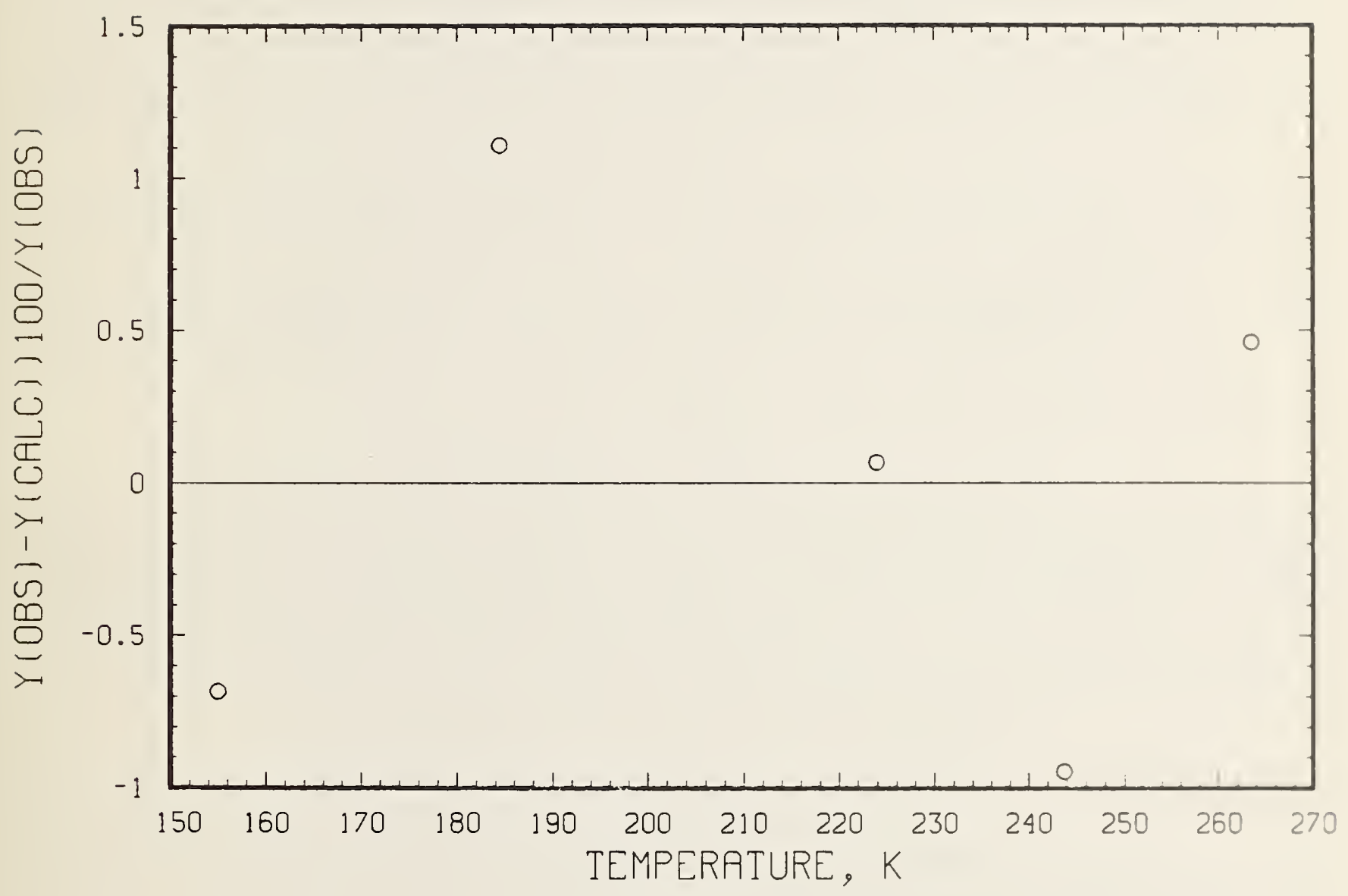

Figure 16. Deviations $(\%)$ between experimental and ritted data as a function of temperature (K) for PMA71-1-vac. 


\section{DISCUSSION}

Heat is conducted through a foamed material by four mechanisms: solid conduction, gas conduction, gas convection, and radiation. The presence of the two nonconductive modes, convection and radiation, results in the possibility that the measured thermal conductivity is thickness dependent. Thermal conductivity is an intrinsic property of the material and does not depend on specimen geometry. The data presented here are the apparent thermal conductivities because of the nonconductive modes of heat transfer. With this understanding, the word apparent is generally not included in the remainder of this report.

Skochdopole (1961) has shown that convective heat transfer will not occur in cells whose dimensions are less than about $3 \mathrm{~mm}$. The manufacturer indicates that the cell diameters of the materials tested here are less than $0.5 \mathrm{~mm}$. Nonstatistical characterization using an optical microscope suggests that this is true. The convective mode will therefore be ignored in the remaining discussion. It is important to keep in mind, however, that if cracks develop in the insulation, either due to thermal or mechanical stresses or debonding, convection can become a dominant mode of heat transfer.

Radiant heat flow through foam insulation is due to both direct plate-to-plate transmission and to absorption and reradiation. Resins used for making polyurethane foams (specimen SOFI) are partially transparent in the 2 to $20 \mu m$ range of wavelengths (Skochdopole, 1961). Sections associated with thermal insulations are normally optically thick (thickness》 photon mean free path), however, so that direct transmission will be small. Specific optical properties of the polymethacrylimide foams are unavailable. If each cell is opaque, the conductivity due to radiant heat transfer, $k_{r}$, can be estimated by

$$
k_{r}=40\left(\frac{\varepsilon}{2-\varepsilon}\right) \ell T^{3}
$$

where $\sigma$ is the Stefan-Boltzman constant $\left(5.67 \times 10^{-8} \mathrm{~W} \cdot \mathrm{m}^{-2} \cdot \mathrm{K}^{-4}\right)$, $\varepsilon$ is the emissivity of foam, $l$ is the average cell dimension parallel to heat flow, and $T$ is the average temperature of the specimen.

Heat flow through the solid component of polymeric foams is through the cell struts and the cell membranes. The component of thermal conductivity which is transmitted through the foam is a function of the thermal conductivity of the resin, the density of the foam, and the distribution of the resin within the struts, membranes, and joints. Qualitatively, the conductivity of the resin, an amorphous solid, is a monotonic function of temperature. At temperatures between approximately 100 and $300 \mathrm{~K}$, the dependence is nearly linear:

$$
k_{\text {res }}=A+B T
$$

where kres is the thermal conductivity of the resin. The conductivity of the solid portion of a foam can be expressed as

$$
k_{S}=k_{r e s} C D
$$


where $\mathrm{C}$ is the factor used to account for distribution of the resin within the cellular structure and $D$ is the foam density expressed in terms of the ratio of resin volume to foam volume. As an example of how the resin distribution contributes to $\mathrm{k}_{\mathrm{S}}$, consider the two different cell structures sketched in Eigure 17(a). The macroscopic density of the foams made up of these cell structures could be assumed to be equal, but the conductivities would be expected to vary considerably. The necked-down strut structure would represent a high resistance to heat flow since $\mathrm{k}_{\mathrm{S}}$ is proportional to the cross section perpendicular to the heat flow (eq 1). The effective path length parallel to the direction of the heat flow is a function of the cell geometry and cell orientation and also contributes to the observed thermal conductivity. Figure 17 (b) illustrates the path length variability both as applied to two foams of the same density and as applied to heat flow parallel to different axes of a single foam. The observed conductivity is inversely proportional to the effective path length (eq 1).

The remaining mode of heat transfer is conduction through the gas occupying the foam cells. When a mixture of gases is present, the conductivity, $k_{g}$, can be estimated by

$$
k_{g}=k_{\text {mixture }}=x_{i=1}^{n} x_{i} k_{i}
$$

where thermal conductivities of the individual species are given by $k_{i}, X_{i}$ represents the corresponding mole fraction, and $n$ is the number of species present. The $k_{i}$ are temperature dependent and are nearly linear functions of temperature. Total gas conduction is given by

$$
k_{g}=X_{C C 1}{ }_{3}{ }^{k_{C C} 1_{3} F}+\left(1-X_{C C 1}{ }_{3}\right) k_{\text {air }}
$$

for cell gas consisting of air and CCl $3 \mathrm{~F}$. Harding (1964) indicates that the values predicted by this simple molar mixing approach tend to be slightly higher than those computed using kinetic theory (Tsederberg, 1965).

Although it is not rigorously correct to add the modes of heat transfer (they are not independent of one another), the total apparent conductivity, kT, obtained using this approximation is acceptable for insulating foams (Gorring and Churchill, 1961):

$$
k_{T}=k_{g}+k_{S}+k_{r}
$$

This $\mathrm{K}_{\mathrm{T}}$ approximates the apparent thermal conductivities determined by ihe research reported here. Quantitative application of the principles discusand above to the experimental results is not possible because of the larf of characterization data for the specimens. They can be used, however, to sugaph. qualitative explanations for the observed differences in the flve specimen?. Th. composite thermal conductivity as a function of temperature for all teats as shown in figure 18 . 


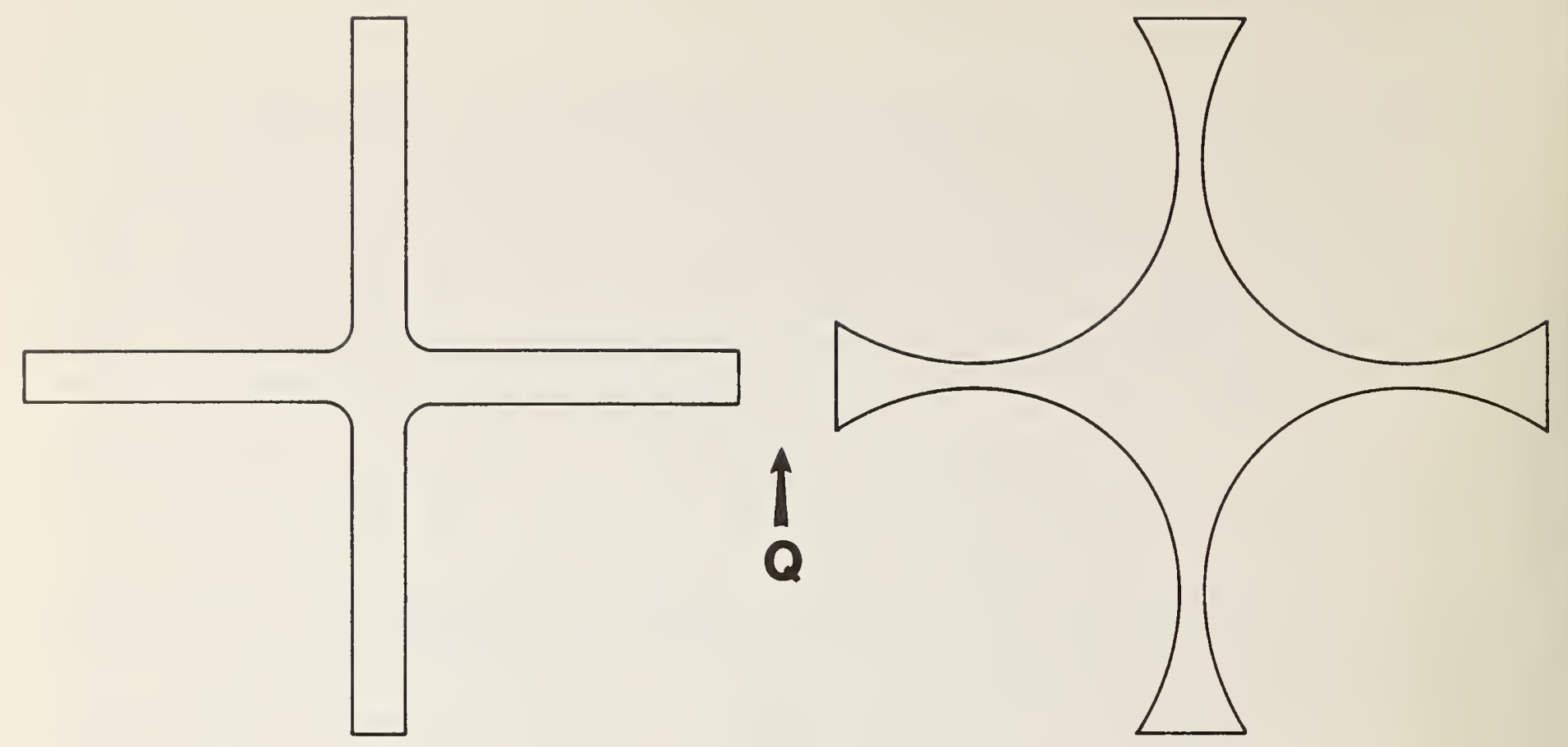

(a)

(b)

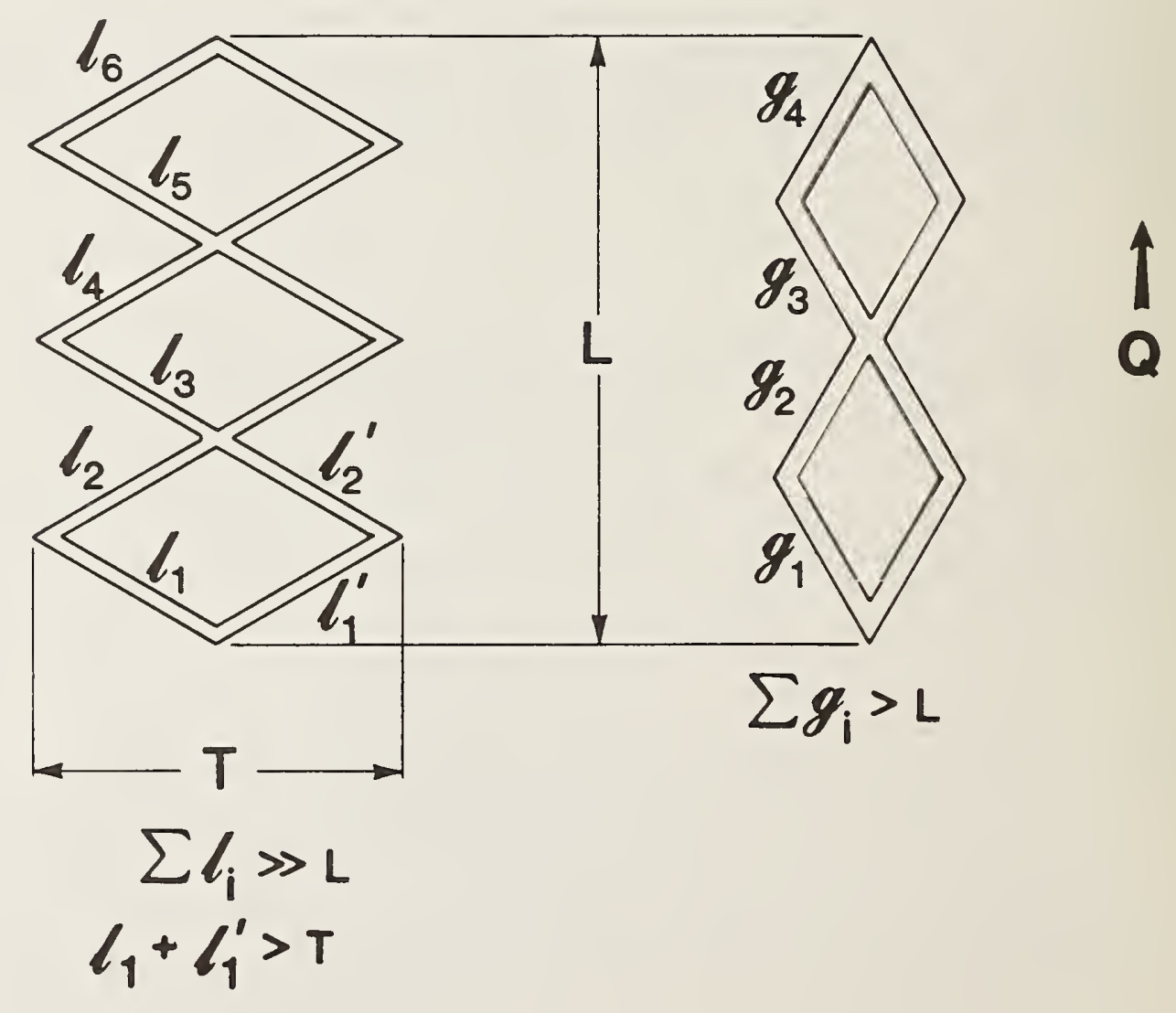

Figure 17. (a) Possible variations of resin distribution in two foams of equal density. (b) possible heat-flow path lengths as a function of cell geometry for two foams of equal density and for different directions in a single foam. 
O so: :

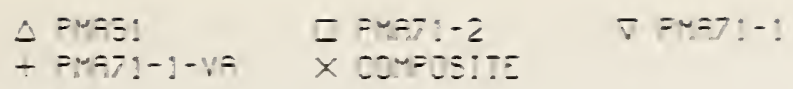

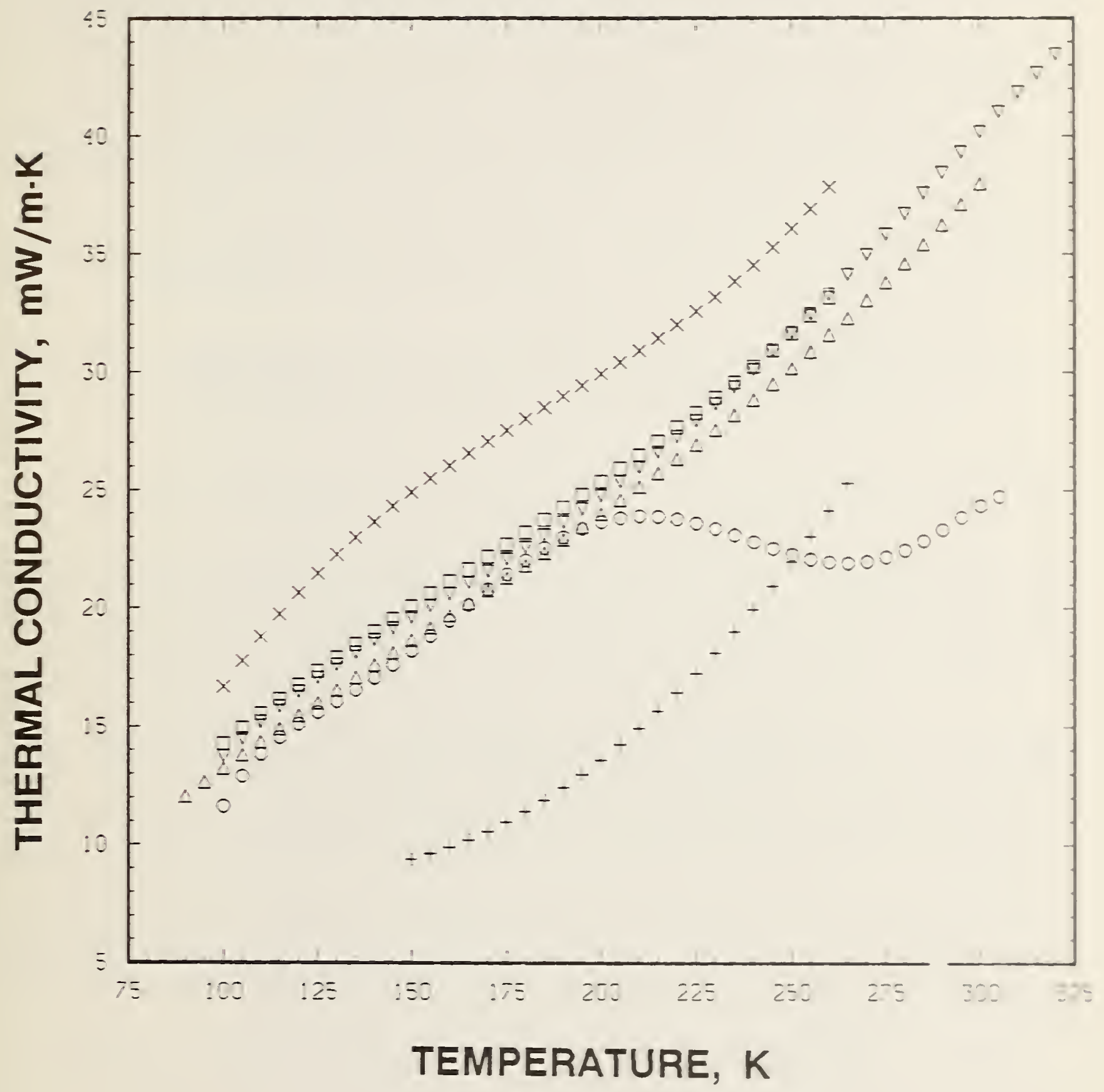

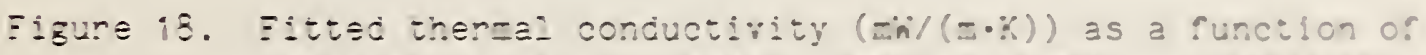

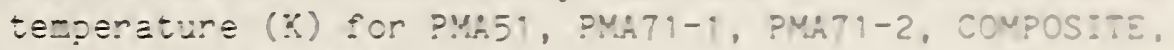
SOEI (PU), and Pld 71-i-vac. 
Attempts to increase our knowledge of the PMA materials by inquiries to the manufacturer were only partially successful due to proprietary restrictions. Literature made available by the distributor indicates that rapid diffusion of air and fill gas occurs. Pending further information from the manufacturer or an in-house characterization, it is only possible to establish circumstantial reasons for the observed thermal conductivity relationships.

Polymethacrylimide series: Specimens with nominal densities of $51 \mathrm{~kg} / \mathrm{m}^{3}$ (PMA51) and $71 \mathrm{~kg} / \mathrm{m}^{3}$ (PMA71-1 and PMA71-2) were tested in dry nitrogen gas and PMA71-1 was also tested at a pressure of approximately $0.67 \mathrm{~Pa}(5 \mu \mathrm{m}$ of $\mathrm{Hg}$ ).

The observed conductivities of the PMA specimens would be expected to be a function of the densities provided the following assumptions are true:

(1) the resin is identical in each foam,

(2) the cell shape, not necessarily size, is the same in each foam,

(3) the distribution of resin is identical in each foam, and

(4) the cell gas, both type and pressure, is the same in each foam.

Based on the experimental results, the most likely scenario is as follows: (a) The gas in the cells is the same for all three specimens; (b) the gas is air; and (c) the assumptions (1), (2), and (3) are essentially true.

The following argument is the basis for the conclusion that the gas in the cells of each foam is air. $k_{g}$ can be expected to account for roughly $50-60 \%$ of the conduction if the cell gas is made up of heavy molecules, such as one of the fluorocarbons or isopropanol, and 60-80\% if the cell gas is air. The difference in thermal conductivity of air and a heavy gas is on the order of a factor of 2.

As discussed later, $\mathrm{kg}$ (SOFI), below about $200 \mathrm{~K}$, is due to air. Below this temperature, $\mathrm{k}_{\mathrm{T}}$ for SOFI and PMA51 are essentially the same. The slopes of the thermal conductivity data below $200 \mathrm{~K}$ suggest that $\mathrm{kg}_{\mathrm{g}}$ for all PMA and SOFI is due to the same gas. The solid conduction component of PMA51 must also be essentially equal to that of SOFI below $200 \mathrm{~K}$. Since $\mathrm{k}_{\mathrm{S}}$ is expected to be a linear function of temperature, $k_{S}$ of SOFI and PMA51 should also be nearly equal at $300 \mathrm{~K}$.

From previous work relating density and thermal conductivity for polyurethane foams (Sparks and Arvidson, 1985), $\mathrm{k}_{\mathrm{S}}$ for SOFI is $11 \mathrm{~mW} /(\mathrm{m} \cdot \mathrm{K})$ and $\mathrm{kg}$ is $13 \mathrm{~mW} /(\mathrm{m} \cdot \mathrm{K})$. Applying the factor of 2 for the increased conductivity of air relative to the fluorocarbon results in $k_{g}(P M A 51)=26 \mathrm{~mW} /(\mathrm{m} \cdot \mathrm{K})$

$$
\begin{aligned}
k_{T}(\text { PMA51) } & \equiv k_{g}+k_{S} \\
& =26+11 \\
& =37 \mathrm{~mW} /(\mathrm{m} \cdot \mathrm{K})
\end{aligned}
$$

This is in agreement with the experimental value. 
If the assumptions that the cells of the PMA specimens contain air are correct, the differences in observed conductivities among PMA51, PMA71-1, and PMA71-2 are due primarily to $\mathrm{k}_{\mathrm{S}}$. As indicated in eq $5, \mathrm{k}_{\mathrm{S}}$ is related to the combined effects of density, resin distribution, and molecular structure of the resin.

$k_{r}$ also contributes to the heat transfered through the foams, but experience with polyurethane foams indicates that this contribution differs by only about 5\% over the range of densities tested here. Other factors being equal, $k_{r}$ would tend to increase the conductivity of PMA51 relative to the more dense specimens.

Figure 19 shows the density dependence of $\mathrm{k}_{\mathrm{T}}$ with temperature as a parameter. These data were obtained from the polynomial relationships for PMA51, PMA71, and COMPOSITE. The rationale for including data for the composite specimen in this figure is given below.

COMPOSITE: The cross section of the composite specimen is shown in figure 20. The thermal conductivity of this specimen should be dominated by the PMA 110 components. The thermal resistances, $R=\Delta X / k_{T}$, of the other components at $250 \mathrm{~K}$ are

$$
\begin{aligned}
R(\text { polyimide film }) & =0.0004\left(\mathrm{~m}^{2} \cdot \mathrm{K}\right) / \mathrm{W} \\
\mathrm{R}(\text { aluminum }) & =8 \times 10^{-7}, \\
\mathrm{R}(\text { adhesive }) & =0.0009 \\
\mathrm{R}(\text { COMPOSITE }) & =1.36
\end{aligned}
$$

The computed resistance balance is

$$
\begin{aligned}
R(\text { COMPOSITE })= & R(\text { PMA110) }+ \\
& {[R(\text { polyimide film })+R(\text { aluminum })} \\
& +3 \times R(\text { adhesive })] \\
= & R(\text { PMA 110)+[0.0031] }
\end{aligned}
$$

so that $R($ COMPOSITE $) \equiv R(P M A 110)$.

The thickness of the upper and lower PMA components of the composite specimen were determined to be 2.3846 and $2.3702 \mathrm{~cm}$ respectively using an optical microscope. Table 25 was computed using the relationship given in eq (9)

$$
k(\text { PMA 110) }=\text { thickness (PMA110) } \times k \text { (COMPOSITE)/thickness (COMPOSITE) }
$$




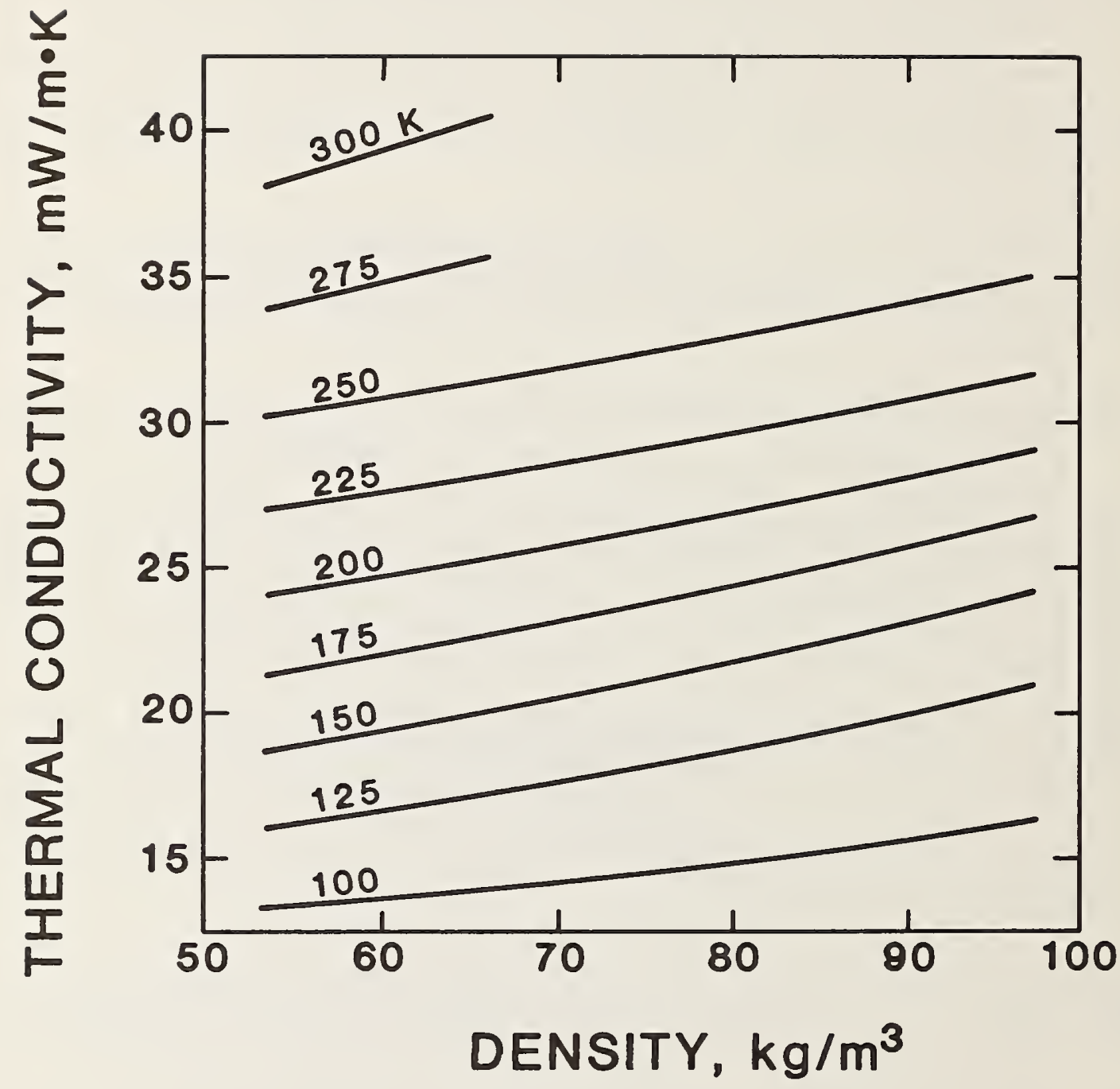

Figure 19. Thermal conductivity $(\mathrm{mW} /(\mathrm{m} \cdot \mathrm{K}))$ as a function of density $\left(\mathrm{kg} / \mathrm{m}^{3}\right)$ with temperature $(K)$ as a parameter for PMA foams. 
毫

E

范

$\frac{2}{5}$

(1)

$\frac{1}{0}$

$\begin{array}{ll}\frac{1}{6} & 0 \\ 0 & 0 \\ \frac{1}{0} & 0 \\ \frac{1}{c} & 0\end{array}$

$\leqslant$ ن $\frac{5}{0} \quad 0$

흥 1

क 5

$\frac{\pi}{5}$

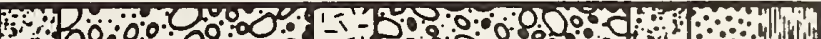

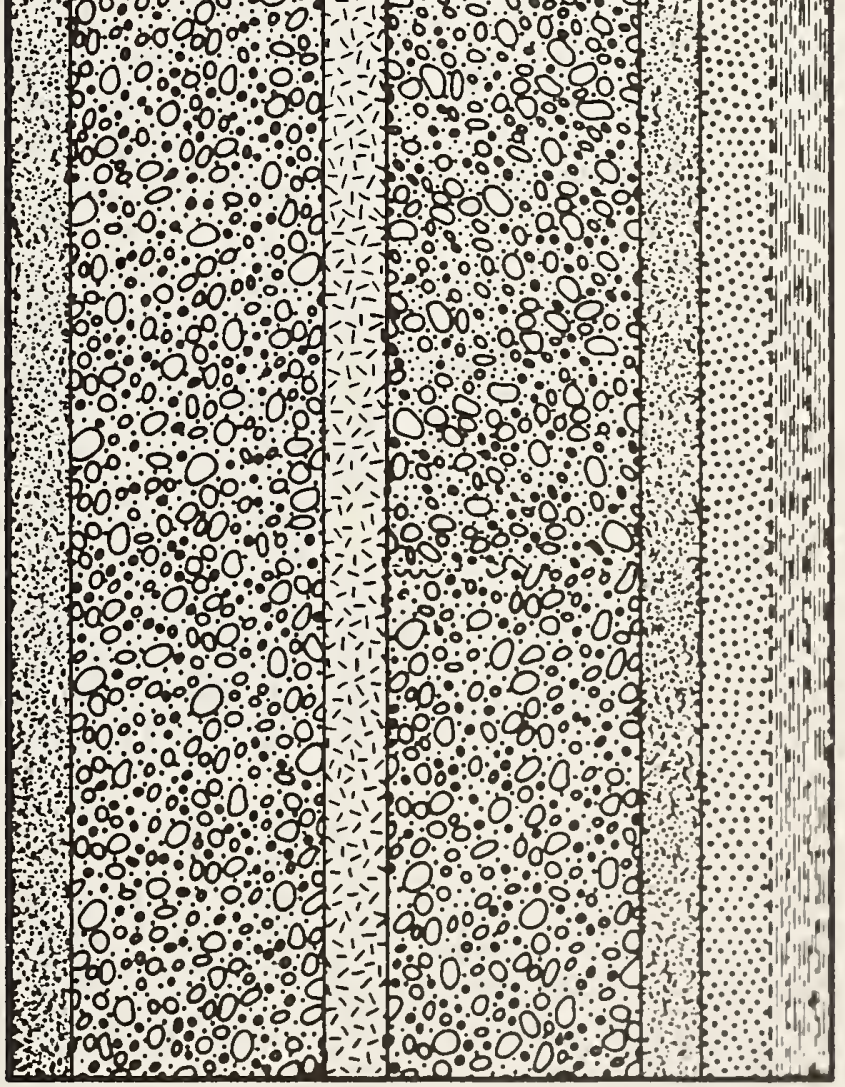


The computed value of $K(P M A 110)$ may include (1) a thickness effect not present in the values for the other PMA specimens if the aluminum-filled adhesive near the center of the composite specimen does not provide an effective radiation shield, or (2) if the adhesive is an effective radiation shield, it would have the net effect of increasing the observed thermal resistance over that for a similar construction where there is no radiation shield. Figure 21 indicates in a general way that the conductivity determined using a thick specimen, e.g., no radiation shield, would be higher than that for a thin specimen. In this case the values given in table 25 would be higher than if they had been determined experimentally on specimens similar in thickness to the other PMA specimens. If the adhesive does serve as an effective radiation shield, however, the values computed for $k$ (PMA110) in table 25 would be lower than would be experimentally determined for specimens similar to the other PMA specimens. Pending further information regarding the emissivity of the aluminum filled adhesive, no estimate of the shielding effectiveness can be made. In either case the errors introduced into the data given in table 25 should not exceed about $\pm 5 \%$ since this is the approximate value expected for the radiative component. The density of the PMA110 component of the composite specimen was determined to be $97.5 \mathrm{~kg} / \mathrm{m}^{3}(6.1$ $\left.1 b / f^{3}\right)$.

SOFI: The thermal conductivity was experimentally determined for the foam/aluminum composite. Data are presented for both the composite and for the foam component. The foam component data were computed using literature values for the conductivity of aluminum 2219-T87. The experimentally determined density of the sprayed polyurethane foam was $37.6 \pm 1.4 \mathrm{~kg} / \mathrm{m}^{3}\left(2.3 \mathrm{lb} / \mathrm{ft}^{3}\right)$.

The thermal conductivity data shown in figures 11 and 13 exhibit the expected temperature dependence for an expanded plastic with a fluorocarbon fill gas. The characteristic curve results when the gas conduction component of the thermal conductivity changes as the mole fraction of heavy gas decreases due to heavy-gas condensation. Equations (6) and (7) can be used to relate the following argument to the observed temperature dependence: (1) At temperatures above about $264 \mathrm{~K}$, the gas conduction is dominated by the unsaturated fluorocarbon gas. Its conductivity is approximately one half that of air, which is the other gas present. (2) Between 264 and $212 \mathrm{~K}$ the fluorocarbon has reached saturation conditions and a vapor-liquid mixture exists in the cells. $\mathrm{kg}$ increases as the mole percent of the fluorocarbon gas decreases. (3) Below $212 \mathrm{~K}$ essentially all of the fluorocarbon has condensed and $\mathrm{kg}$ is entirely due to air.

The polynomial fitting procedure used to represent the experimental data for this specimen is not the optimum for this complicated function of temperature. This is evident from the deviation plots given in figures 12 and 14: the deviations are about a factor of two greater than those for the PMA specimens. The inability to accurately represent the experimental data is also evident in figures 11 and 13. The analytical minimum at $264 \mathrm{~K}$ is too high by $2 \%$ and an artificial wiggle at about $140 \mathrm{~K}$ causes the analytical value to be too 10 w by $2 \%$. The rapid decrease in conductivity below $110 \mathrm{~K}$, figure 18 and tables 18 and 21 , is also an artifact created by extrapolation of a polynomial. 


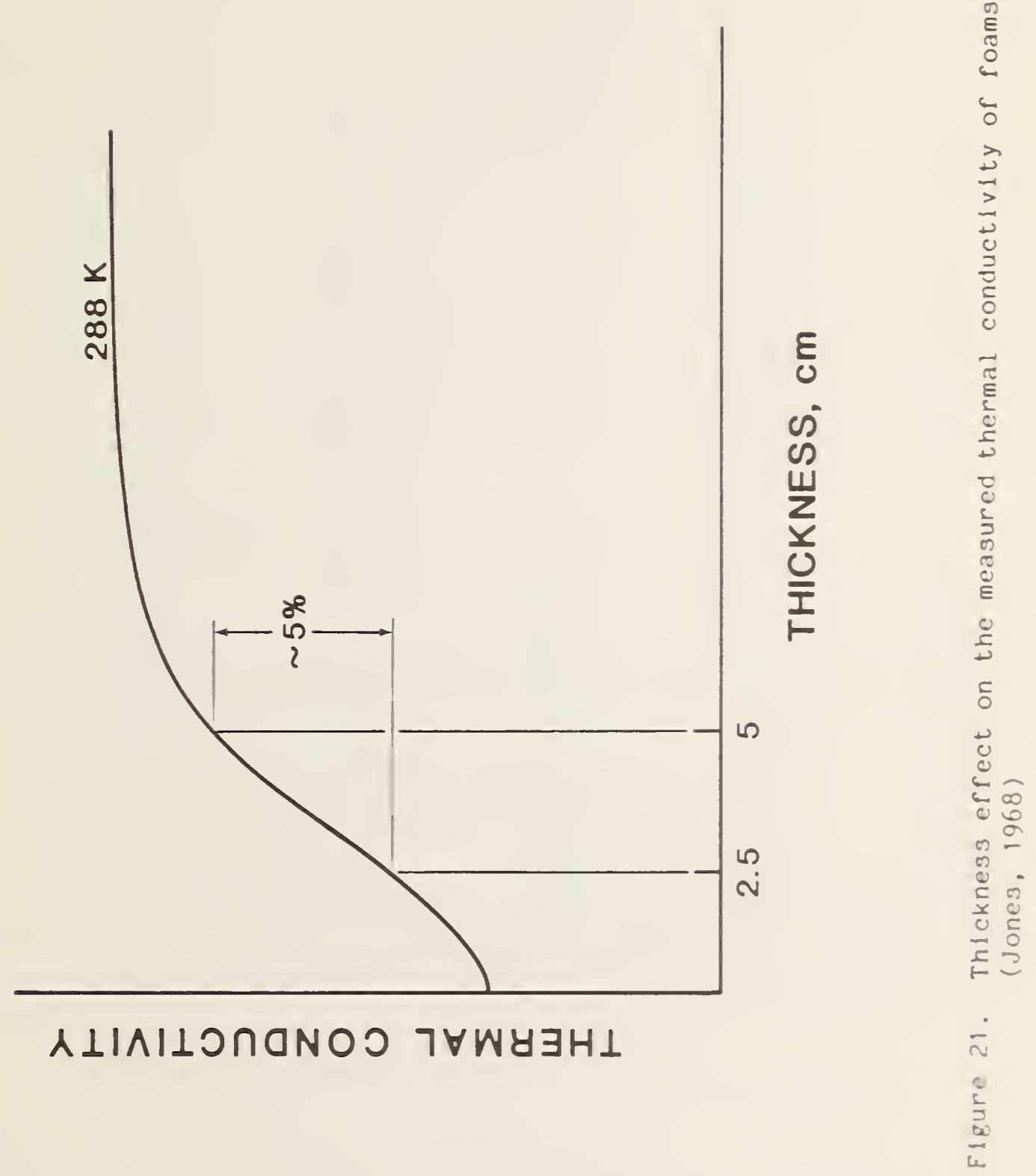


Table 25. Computed thermal conductivity $(\mathrm{mW} /(\mathrm{m} \cdot \mathrm{K}))$ as a function of temperature $(K)$ for a polymethacrylimide foam of density $97.5 \mathrm{~kg} / \mathrm{m}^{3}\left(6.1 \mathrm{lb} / \mathrm{ft}^{3}\right)$.

$\begin{array}{cccccc}T & k & T & k & T & k \\ 100.0 & 16.17 & 154.0 & 24.61 & 208.0 & 29.77 \\ 102.0 & 16.61 & 156.0 & 24.82 & 210.0 & 29.97 \\ 104.0 & 17.03 & 158.0 & 25.04 & 212.0 & 30.17 \\ 106.0 & 17.44 & 160.0 & 25.25 & 214.0 & 30.38 \\ 108.0 & 17.84 & 162.0 & 25.45 & 216.0 & 30.59 \\ 110.0 & 18.23 & 164.0 & 25.65 & 218.0 & 30.80 \\ 112.0 & 18.61 & 166.0 & 25.85 & 220.0 & 31.02 \\ 114.0 & 18.97 & 168.0 & 26.04 & 222.0 & 31.24 \\ 116.0 & 19.33 & 170.0 & 26.24 & 224.0 & 31.47 \\ 118.0 & 19.68 & 172.0 & 26.43 & 226.0 & 31.70 \\ 120.0 & 20.02 & 174.0 & 26.62 & 228.0 & 31.94 \\ 122.0 & 20.35 & 176.0 & 26.80 & 230.0 & 32.18 \\ 124.0 & 20.67 & 178.0 & 26.99 & 232.0 & 32.43 \\ 126.0 & 20.98 & 180.0 & 27.17 & 234.0 & 32.69 \\ 128.0 & 21.29 & 182.0 & 27.35 & 236.0 & 32.95 \\ 130.0 & 21.58 & 184.0 & 27.54 & 238.0 & 33.21 \\ 132.0 & 21.87 & 186.0 & 27.72 & 240.0 & 33.49 \\ 134.0 & 22.15 & 188.0 & 27.90 & 242.0 & 33.77 \\ 136.0 & 22.43 & 190.0 & 28.08 & 244.0 & 34.06 \\ 138.0 & 22.69 & 192.0 & 28.26 & 246.0 & 34.36 \\ 140.0 & 22.95 & 194.0 & 28.45 & 248.0 & 34.67 \\ 142.0 & 23.21 & 196.0 & 28.63 & 250.0 & 34.98 \\ 144.0 & 23.45 & 198.0 & 28.82 & 252.0 & 35.30 \\ 146.0 & 23.70 & 200.0 & 29.00 & 254.0 & 35.64 \\ 148.0 & 23.93 & 202.0 & 29.19 & 256.0 & 35.98 \\ 150.0 & 24.16 & 204.0 & 29.38 & 258.0 & 36.33 \\ 152.0 & 24.39 & 206.0 & 29.58 & & \end{array}$


PMA71-1-vac: The low-pressure data in this experimental set were obtained using the same specimens as were tested earlier at ambient pressures. After reinstalling the specimens in the apparatus, an ambient pressure data point was taken to assure that no undue specimen changes had. occured and that the reinstallation itself did not introduce significant differences in the measured conductivity. The result of the new, ambient-pressure data point was $\mathrm{k}_{\mathrm{T}}=33.32 \mathrm{~mW} /(\mathrm{m} \cdot \mathrm{K})$ at $263.525 \mathrm{~K}$. This is within $1.6 \%$ of the previous data and is within the combined random uncertainty of the two data sets.

As seen in figure 18, the thermal conductivity of the PMA71-1-vac, determined in a vacuum environment of $0.67 \mathrm{~Pa}(5 \mu \mathrm{m}$ of $\mathrm{Hg})$, is significantly lower than that for the same specimens in an ambient-pressure, dry-nitrogen environment. The temperature dependence of the conductivity is also different. These observed differences are assumed to be due to the change in the thermal contact between the GHP plates and the specimens. In the nitrogen environment heat is transfered between the plates and the specimen by solid and gaseous conduction and radiation. The vacuum environment reduces the gas conduction component. The temperature drop across the boundary layer created by cut and damaged cells is shown schematically in figure 22 and can be computed using eq (10).

$$
\Delta \mathrm{T}_{\text {Boundary }}=Q_{\text {total }} \frac{\Delta \mathrm{X}_{\mathrm{B}}}{\text { kresin }_{\text {resin }}+4 \sigma E \mathrm{~T}^{3} \Delta \mathrm{X}_{\mathrm{B}} \mathrm{A}_{\text {vacuum }}}
$$

In order to determine the temperature drop across the boundary layer, $\Delta X_{B}$, several parameters shown in eq (10) must be known: kresin, the conductivity of the resin; the emissivities of the resin and the apparatus plates,

$$
E=\frac{\varepsilon_{\text {plate }} \varepsilon_{\text {resin }}}{\varepsilon_{\text {plate }}+\varepsilon_{\text {resin }}-\varepsilon_{\text {plate }} \varepsilon_{\text {resin }}},
$$

contact areas of the resin, Aresin, and the vacuum, Avacuum; and the effective thickness of the layer of broken or damaged cells, $\Delta X_{B}$. A quantitative evaluation of eq (10) cannot be made without knowledge of the above parameters. It is possible, however, to separate the overall boundary resistance from that of the bulk foam since

$$
\begin{aligned}
R_{\text {observed, vacuum }} & =R_{\text {boundary, cold }}+R_{\text {PMA71-1 }}+R_{\text {boundary, hot }} \\
& =R_{\text {PMA71-1 }}+2 R_{B}
\end{aligned}
$$

Table 26 gives the average boundary resistances computed using this macroscoplc relationship (approximate since the resistance of the boundary at the cold plate would be larger than that at the hot plate). 


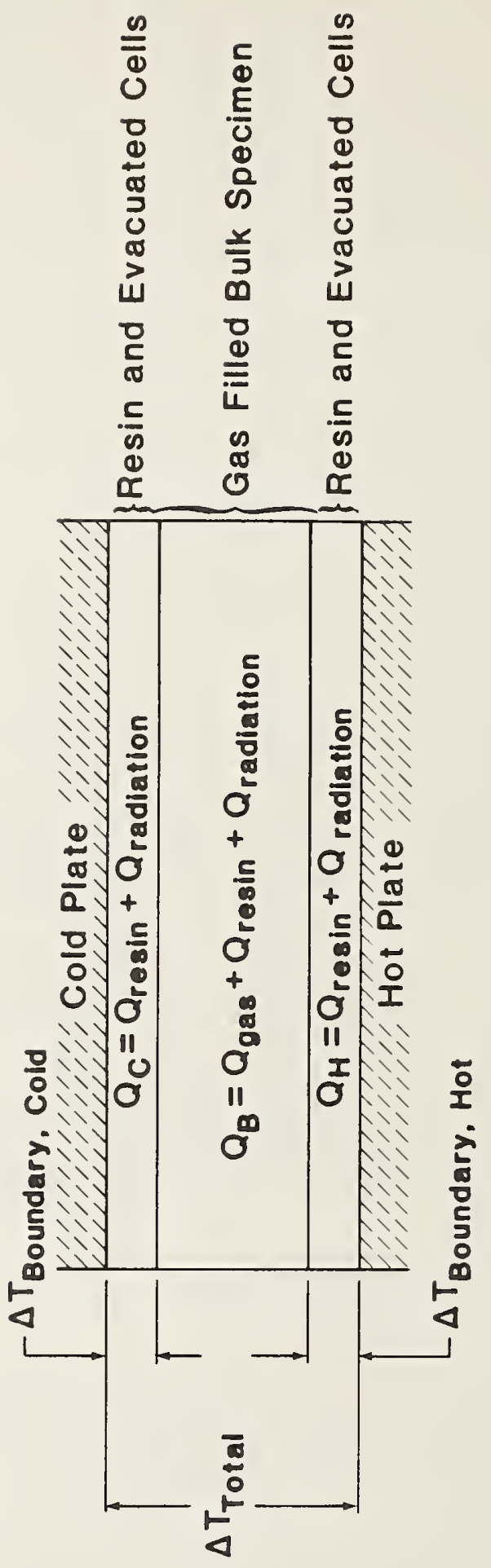

J

究

$\frac{5}{2}$

文

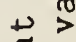

幽

प 吾

a

के

든

(1)

30

\%

ฮ

응 응

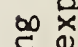

it

ชิ

효

0
$\rightarrow$
0

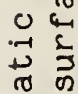

离

$\dot{N}$
0
0
0
0
0 
Table 26. Thermal resistance due to evacuated boundaries, $R_{B}$, for PMA71-1.

$\begin{array}{cccccc}\text { TEMPERATURE } & \mathrm{R} \text { (gas) } & \mathrm{R} \text { (vacuum) } & \Delta \mathrm{R} & \mathrm{R}_{\mathrm{B}} & 2 \mathrm{R}_{\mathrm{B}} / \mathrm{R} \text { (vacuum) } \\ (\mathrm{K}) & \left(\left(\mathrm{m}^{2} \cdot \mathrm{K}\right) / \mathrm{W}\right) & \left(\left(\mathrm{m}^{2} \cdot \mathrm{K}\right) / \mathrm{W}\right) & \left(\left(\mathrm{m}^{2} \cdot \mathrm{K}\right) / \mathrm{W}\right) & \left(\left(\mathrm{m}^{2} \cdot \mathrm{K}\right) / \mathrm{W}\right) & (\%) \\ 150 & 1.30 & 2.73 & 1.43 & 0.72 & 53 \\ 200 & 1.03 & 1.88 & 0.85 & 0.42 & 45 \\ 250 & 0.81 & 1.17 & 0.36 & 0.18 & 31\end{array}$

The boundary resistances are seen to represent a significant portion of the observed overall resistance and become increasingly dominant as the temperature decreases.

\section{SUMMARY}

The thermal conductivity of five thermal insulations was determined in the approximate temperature range from 100 to $300 \mathrm{~K}$. Three specimens were expanded polymethacrylimide and another was a sandwich construction utilizing a more dense polymethacrylimide foam. The fifth specimen was a composite made by spraying polyurethane foam onto an aluminum substrate. All tests were conducted in a guarded-hot-plate apparatus. The environment for the tests was ambient pressure nitrogen gas with one exception: one PMA specimen was retested at $0.67 \mathrm{~Pa}$ (5 $\mu \mathrm{m}$ of $\mathrm{Hg}$ ).

The results for the PMA tests indicate density dependences which are nominally expected for most expanded plastics. The straight-line temperature dependence of these materials indicates that the unknown cell gas does not condense in the temperature range investigated or that its conductivity is near that of air. By contrast, the data for the polyurethane specimen show a temperature dependence typical for condensation of a heavy gas component in an air/heavy-gas mixture.

The vacuum environment data suggest an unexpectedly strong effect due to the foam layer made up of broken cells. Not only is the magnitude of the conductivity reduced significantly, but the form of the temperature dependence is also affected.

Characterization of the specimens was limited to density. Without knowledge of parameters such as resin conductivity, species and pressures of gas present in the cells, cell size, shape, and orientation, a quantative determination of the contribution of the various modes of heat transfer cannot be made. 


\section{REFERENCES}

ASTM. Annual Book of Standards. American Society for Testing and Materials, Philadelphia, PA, Standard C177, Steady-State Thermal Transmission Properties by Means of the Guarded Hot Plate, Part 18.

Gorring, R. L. and Churchill, S. W. (1961), "Thermal Conductivity of Heterogeneous Materials," Chem. Eng. Prog., 57, 53-59.

Harding, R. H. (1964), "Heat Transfer Through Low-Density Cellular Materials," I and EC Process Design and Development, 3, 117-125.

Jones, T. T. (1968), "Effect of thickness and temperature on Heat Transfer through Foamed Polymers", Proceedings of the Seventh Thermal Conductivity Conference, NBS Spec. Publ. 302, 737-748.

Skochdopole, R.E. (1961), "The Thermal Conductivity of Foamed Plastics," Chem. Eng. Prog., 57, 55-59.

Smith, D. R., Hust, J. G., and Van Poolen, L. J. (1982), "A Guarded-Hot-Plate Apparatus for Measuring Effective Thermal Conductivity of Insulations Between $80 \mathrm{~K}$ and $360 \mathrm{~K}, "$ NBSIR 81-1657, National Bureau of Standards, Boulder, CO.

Somers, E. V. and Cyphers, J. A. (1951), "Analysis of Errors in Measuring Thermal Conductivity of Insulating Materials," Rev. Sci. Instrum., 22, 583-586.

Sparks, L. L. and Arvidson, J. M. (1985), "Thermal and Mechanical Properties of Polyurethane Foams at Cryogenic Temperatures", J. of Thermal Insulation, 8, $198-232$.

Tsederberg, N. V. (1965), Thermal Conductivity of Gases and Liquids, Cambridge, MA, MIT Press, p. 144.

Tye, R. P. and Spinney, S. C. (1976), "Thermal Conductivity of Concrete: Measurement Problems and Effect of Moisture," Bull. Int. Froid, Annexe 1976-2, 119-127. 
NBS.114A REV. 2.8C)

U.S. DEPT. OF COMM.

BIBLIOGRAPHIC DATA

SHEET (See instructions)

1. PUBLICATION OR REPORT NO.

NBSIR $88-3086$

2. Performing Organ. Report No.

3. Publication Date

January 1988

4. TITLE AND SUBTITLE

Thermal Conductivity of Selected Foams and Systems from 100 to $300 \mathrm{~K}$

5. AUTHOR(S)

L. L. Sparks, W. P. Dubé, and A. J. Slifka

6. PERFORMING ORGANIZATION (If joint or other than NBS. see instructions)

NATIONAL BUREAU OF STANDARDS

DEPARTMENT OF COMMERCE

WASHINGTON, D.C. 20234

7. Contract/Grant No.

L85254B

8. Type of Report \& Period Covered

Fina1, May-July 1986

9. SPONSORING ORGANIZATION NAME AND COMPLETE ADDRESS (Street, City, State, ZIP)

NASA Langley Research Center

Hampton, VA 23665

10. SUPPLEMENTARY NOTES

[] Document describes a computer program; SF-185, FIPS Software Summary, is attached.

11. ABSTRACT (A 200-word or less factual summary of most significant information. If document includes a significant bibliography or literature survey. mention it here)

The apparent thermal conductivity of five insulating materials was determined in the nominal temperature range from 100 to $300 \mathrm{~K}$ $(-280$ to $80 \mathrm{oF})$. A guarded-hot-plate apparatus was used and the testing environment was dry nitrogen gas at near ambient pressure. One specimen was retested in a vacuum environment.

Three of the specimens were neat polymethacrylimide (PMA) foams with nominal densities of 51 and $71 \mathrm{~kg} / \mathrm{m}^{3}\left(3.2\right.$ and $\left.4.41 \mathrm{~b} / \mathrm{ft}^{3}\right)$. Other specimens tested were a polyurethane foam sprayed on an aluminum substrate and a sandwich construction specimen utilizing PMA foam of nominal density $110 \mathrm{~kg} / \mathrm{m}^{3}\left(6.9 \mathrm{lb} / \mathrm{ft}^{3}\right)$. The results in nitrogen gas show the expected conductivity increase with increasing density. The observed conductivity at $0.67 \mathrm{~Pa}(5 \mu \mathrm{m}$ of $\mathrm{Hg}$ ) is significantly lower than that in ambient-pressure nitrogen gas. The large relative difference may be due to increased thermal resistance at the plate-specimen boundaries.

12. KEY WORDS (Six to twelve entries; alphabetical order; capitalize only proper names; and separate key words by semicolons) foam; insulation; low-temperature; polymethacrylimide;

polyurethane; thermal conductivity.

13. AVAILABILITY

X] Unlimited

$\square$ For Official Distribution. Do Not Release to NTIS

$\square$ Order From Superintendent of Documents, U.S. Government Printing Office, Washington, D.C. 20402.

X] Order From National Technical Information Service (NTIS), Springfield, VA. 2216I
14. NO. OF

PRINTED PAGES

60

15. Pilce 




